\title{
The lifetimes of planetary debris discs around white dwarfs
}

\author{
Dimitri Veras ${ }^{1,2 \star} \dagger$, Kevin Heng ${ }^{2,3}$ \\ ${ }^{1}$ Centre for Exoplanets and Habitability, University of Warwick, Coventry CV4 7AL, UK \\ ${ }^{2}$ Department of Physics, University of Warwick, Coventry CV4 7AL, UK \\ ${ }^{3}$ Center for Space and Habitability, University of Bern, Gesellschaftsstrasse 6, CH-3012 Bern, Switzerland
}

8 June 2020

\begin{abstract}
The lifetime of a planetary disc which orbits a white dwarf represents a crucial input parameter into evolutionary models of that system. Here we apply a purely analytical formalism to estimate lifetimes of the debris phase of these discs, before they are ground down into dust or are subject to sublimation from the white dwarf. We compute maximum lifetimes for three different types of white dwarf discs, formed from (i) radiative YORP breakup of exo-asteroids along the giant branch phases at $2-100 \mathrm{au}$, (ii) radiation-less spin-up disruption of these minor planets at $\sim 1.5-4.5 R_{\odot}$, and (iii) tidal disruption of minor or major planets within about $1.3 R_{\odot}$. We display these maximum lifetimes as a function of disc mass and extent, constituent planetesimal properties, and representative orbital excitations of eccentricity and inclination. We find that YORP discs with masses up to $10^{24} \mathrm{~kg}$ live long enough to provide a reservoir of surviving $\mathrm{cm}$-sized pebbles and $\mathrm{m}$ - to $\mathrm{km}$-sized boulders that can be perturbed intact to white dwarfs with cooling ages of up to 10 Gyr. Debris discs formed from the spin or tidal disruption of these minor planets or major planets can survive in a steady state for up to respectively $1 \mathrm{Myr}$ or $0.01 \mathrm{Myr}$, although most tidal discs would leave a steady state within about 1 yr. Our results illustrate that dust-less planetesimal transit detections are plausible, and would provide particularly robust evolutionary constraints. Our formalism can easily be adapted to individual systems and future discoveries.
\end{abstract}

Key words: Kuiper belt: general minor planets, asteroids: general planets and satellites: dynamical evolution and stability stars: evolution white dwarfs protoplanetary discs.

\section{INTRODUCTION}

The death throes of planetary systems represent unique tracers of their evolutionary history. Giant branch host stars dynamically excite their planetary constituents, resulting in gravitational instabilities which eventually manifest themselves as detectable debris close to and within the photosphere of the eventual white dwarfs, where the chemical constituents of exo-planetesimals can be measured.

As a host star leaves the main sequence, it will expand its radius by a factor of hundreds, shed over half of its mass, and increase its luminosity by factor of thousands. All orbiting objects, including both major and minor planets, migrate outward due to the stellar mass loss (Omarov 1962; Hadjidemetriou 1963; Veras et al. 2011), but may fail to "outrun" the enlarging star and be engulfed (Kunitomo et al. 2011; Mustill \& Villaver

\footnotetext{
* E-mail: d.veras@warwick.ac.uk

$\dagger$ STFC Ernest Rutherford Fellow
}

2012; Adams \& Bloch 2013; Nordhaus \& Spiegel 2013; Villaver et al. 2014; Madappatt et al. 2016; Staff et al. 2016; Gallet et al. 2017; Rao et al. 2018; Sun et al. 2018; Ronco et al. 2020). Exo-planetary analogues to Mars, Jupiter, Saturn, Uranus and Neptune are sufficiently separated from their parent stars to typically survive engulfment (Schröder \& Smith 2008; Veras 2016b).

If the surviving major planets are sufficiently "packed" together, then the stellar mass loss could change their stability boundaries (Debes \& Sigurdsson 2002; Veras et al. 2013, 2018; Vovatzis et al. 2013; Mustill et al. 2014; Veras \& Gänsicke 2015). The stability boundaries between minor and major planets are also altered (Bonsor et al. 2011; Debes et al. 2012; Frewen \& Hansen 2014; Petrovich \& Muñoz 2017; Mustill et al. 2018; Smallwood et al. 2018). The resulting gravitational instabilities are rarely triggered immediately, and usually only after the star has become a white dwarf. Although the minor planets themselves might survive engulfment, they struggle to survive intact because the highest luminosities 
achieved during the giant branch phases easily pushes around (Veras et al. 2015a, 2019a) and spins up these bodies, commonly to breakup speed (Veras et al. 2014b; Veras \& Scheeres 2020).

The subsequent significant reservoirs of pebbles, cobbles, boulders, minor planets and major planets which orbit a newly born white dwarf can reside at separations anywhere between a few au and on the order of $10^{2}$ au. This debris disc provides the primary source of mass that can be dynamically excited and perturbed towards the white dwart. If this disc survives in a largely collision-less state on Gyr timescales, then perturbed constituents would arrive at the white dwarf intact. Otherwise, the constituents might have already been pulverized into dust by the time they approach the white dwarf. One focus of our investigation here is to distinguish these possibilities by computing YORP disc lifetimes.

The exo-planetesimals which do reach the immediate region of the white dwarf, within several solar radii 2 , have now been observed in three successive stages of their evolution, as:

(i) Fully or partially intact, sometimes with accompanying fragments, orbiting the white dwarf (Vanderburg et al. 2015; Manser et al. 2019; Vanderbosch et al. 2020),

(ii) Broken up into an annulus of dust and sometimes gas around the white dwarf (Zuckerman \& Becklin 1987; Farihi 2016; Manser et al. 2020), and

(iii) Chemically stratified at the atomic level in the photosphere of the white dwarf (van Maanen 1917, 1919; Zuckerman et al. 2007; Dufour et al. 2010; Klein et al. 2010; Gänsicke et al. 2012; Jura \& Young 2014).

So far the first two stages have been observed concurrently, with dusty effluences and fragment distributions of at least one disintegrating exo-planetesimal that are detectable with photometric transit depths exceeding 50 per cent (Vanderburg \& Rappaport 2018). In the third stage, the white dwarf photosphere reveals exquisite chemical profiles of exo-planetary material at a level of detail exceeding that of any other exoplanetary detection technique (Harrison et al. 2018; Hollands et al. 2018; Doyle et al. 2019; (Swan et al. 2019b; Bonsor et al. 2020).

Linking and understanding the timescales for all of these stages relies on first understanding the lifetime of the debris disc in the first stage. This evolution of this disc, which is the second focus of our study, sets up the subse-

1 More distant sources provide minor contributions. Major planets are not expected to form beyond $10^{2}$ au. In the range $10^{2}-10^{3}$ $\mathrm{au}$, large, Ceres-like minor planets largely survive and retain the same orbits that were set by the primordial stellar cluster evolution Veras et al. 2020b) but are not numerous. Boulders and pebbles could easily be radiatively thrust into this $10^{2}-10^{3}$ au region during the giant branch phases (Veras et al. 2019a), but represent a small total amount of mass. From $10^{3}-10^{4} \mathrm{au}$, inner exo-Oort clouds would survive (Stone et al. 2015; Caiazzo \& Heyl 2017), but comets beyond $10^{4}$ au are thought to be severely depleted due to gravitational escape from giant branch mass loss Veras \& Wvatt 2012; Veras et al. 2014d d).

2 Giant exoplanets themselves can also be perturbed towards the white dwarf, as evidenced by a reported ice giant planet orbiting at an approximate distance of just 0.07 au from WD J0914+1914 Gänsicke et al. 2019; Veras \& Fuller 2019, 2020). quent evolution of the system, where dust and gas is generated, and eventually accreted onto the white dwarf.

The replenishment timescale - how often exoplanetesimals approach, break-up around and deposit themselves into a white dwarf - is one of the most important unknown parameters in post-main-sequence planetary science. Two reasons are

(i) This replenishment timescale is linked to and perhaps constrained by the $25-50$ per cent of the white dwarf population which is "metal polluted" (containing photospheric exoplanetary matter), the 1.5 per cent of white dwarfs which are both metal polluted and contain a dusty disc Wilson et al. 2019), the 0.07 per cent of white dwarfs which are metalpolluted and contain both observable dust and gas in a surrounding disc (Manser et al. 2020), and the observational biases against their detection (e.g. Bonsor et al. 2017).

(ii) Helium-rich white dwarfs can retain accreted exoplanetary material in their convective zones for up to about one Myr, depending on the age of the star. Lower limits for this accumulated mass are detectable (Farihi et al. 2010; Girven et al. 2012; Xu \& Jura 2012). Hence the replenishment timescale, coupled with measured limits on accumulated mass, can place constraints on the deposition or accretion rate onto the white dwarf.

Previous estimates of the replenishment timescale Girven et al. 2012) mix inferred convection zone masses from helium-rich metal-polluted white dwarfs (DBZ spectral type) with accretion rates of hydrogen-rich metal-polluted white dwarfs (DAZ spectral type). This inconsistency motivates alternate approaches, despite having provided a useful, working estimate $\left(\sim 10^{4}-10^{6}\right.$ yr $)$ for nearly a decade.

From theoretical modelling perspectives, identifying the lifetime of different stages of a debris, dusty or gaseous disc has not been a particular focus. Instead, the much shorter formation process of a debris disc has received more attention. Further, for the au-scale debris discs which are produced from giant branch luminosity spin-up of exo-asteroids to breakup speed (Veras et al. 2014b; Veras \& Scheeres 2020) - an effect known as the YORP effect (Rubincam 2000) - the authors did not consider evolution after formation.

For the debris discs generated from exo-planetesimals approaching the white dwarf, there are (at least) two formation channels: tidal disruption within the Roche radius of the white dwarf (Graham et al. 1990; Jura 2003; Debes et al. 2012; Veras et al. 2014a; Malamud \& Perets $2020 \mathrm{a}, \mathrm{b}$ ) and spin disruption outside of the Roche radius (Makarov \& Veras 2019; Veras et al. 2020a). The spin disruption mechanism occurs when the exchange between spin and angular momentum of exo-planetesimals on highly eccentric orbits becomes chaotic, and can spin these objects up to break-up speed. This mechanism applies to aspherical asteroids (most solar system asteroids are aspherical) whose spin rate is altered with each pericentre passage, and can eventually increase to break-up speed.

Most post-formation evolution models of the discs which are produced from tidal disruption have considered the combined effect of gas and dust, or gas only (Bochkarev \& Rafikov 2011; Rafikov 2011a, b; Metzger et al. 2012; Rafikov \& Garmilla 2012; Kenvon \& Bromlev 2017b; Miranda \& Rafikov 2018; O'Connor \& Lai 2020). But what 
of the evolution of the debris discs which are formed beyond the sublimation distance - sometimes well beyond this distance - and in particular for cold white dwarfs? For this gas-free case, Kenyon \& Bromley (2017a) investigated collisional cascades of the debris over $10^{6}$ yr using a sophisticated coagulation and fragmentation code, and incorporated a steady stream of external particles throughout the disc evolution.

Here, we instead apply a version of the entirely analytic formalism of Heng \& Tremaine (2010) to estimate the lifetime of three types of debris discs around white dwarfs: those produced by the YORP effect, those produced from radiation-less rotational disruption (henceforth labelled as "spin" discs), and those produced from tidal disruption; see Fig. 1 for a schematic overview. This formalism, while necessarily imposing some restrictive assumptions, allows us to explore the entire parameter space as a function of disc and planetesimal properties. The results can then be adapted to individual systems.

In Section 2 we justify in detail our numerical choices for the input parameters to the model. Then in Section 3 we illustrate the output quantities from the model, and in some cases slightly generalize the formulae of Heng \& Tremaine (2010). We perform the computations and present our results in Section 4 and then discuss these results in Section 5. We summarize our investigation in Section 6.

\section{GIVEN VARIABLES}

Required inputs into the model of Heng \& Tremaine 2010) include properties from the star, individual planetesimals, and the disc as a whole. We select parameter ranges for each of these variables based on a mixture of observational and theoretical constraints.

\subsection{The star: $M_{\star}$}

From the white dwarf itself, only the mass $M_{\star}$ is required to be input into the formalism of Heng \& Tremaine (2010) and in particular Heng \& Malik (2013), who adopted an arbitrary stellar mass. The vast majority of single white dwarfs harbour masses in the range $0.4-0.8 M_{\odot}$, with a peak around $0.60-0.65 M_{\odot}$; this peak is sharp (Tremblav et al. 2016; Cummings et al. 2018).

From the mass alone, the radius of the white dwarf $R_{\star}$ can be analytically estimated. $R_{\star}$ can be expressed entirely in terms of $M_{\star}$ Nauenberg 1972; Verbunt \& Rappaport 1988)

$$
\frac{R_{\star}}{R_{\odot}} \approx 0.0127\left(\frac{M_{\star}}{M_{\odot}}\right)^{-1 / 3} \sqrt{1-0.607\left(\frac{M_{\star}}{M_{\odot}}\right)^{4 / 3}}
$$

such that a range of $M_{\star}=0.4-0.8 M_{\odot}$ corresponds to $R_{\star}=0.0156-0.0125 R_{\odot}$, or $R_{\star}=7.26 \times 10^{-5}-5.83 \times 10^{-5}$ au.

Other stellar parameters which are important for context but are not necessarily input variables are the white dwarf's temperature $T_{\star}$, luminosity $L_{\star}$ and cooling age $t_{\text {cool }}$. The cooling age is defined as the time since the white dwarf was born (not the time from the birth in the stellar cluster), and is relevant for this paper because disc lifetime might be a function of $t_{\text {cool }}$ due to radiative effects. All three parameters are related non-trivially, and both $T_{\star} \approx 4 \times 10^{3}-1 \times 10^{5} \mathrm{~K}$ and $L_{\star} \approx 10^{2}-10^{-5} L_{\odot}$ are functions of $t_{\text {cool }}$ Mestel 1952; Althaus et al. 2010; Koester 2013). The function is steep: by the time the white dwarf has cooled to $t_{\text {cool }} \approx 10 \mathrm{Myr}$, $T_{\star}$ reduces from its peak value to a value of a few tens of thousands of $\mathrm{K}$, when $L_{\star} \approx 0.1 L_{\odot}$. The oldest known white dwarf planetary systems have $t_{\text {cool }} \approx 8$ Gyr Hollands et al. 2017).

Overall, we adopt the range $M_{\star}=0.4-0.8 M_{\odot}$.

\subsection{The planetesimals: $M_{\mathrm{p}}, R_{\mathrm{p}}, \rho_{\mathrm{p}}$}

Properties of the planetesimals are more uncertain. We assume that the planetesimals which comprise the disc are spheres and have masses, radii and densities which are denoted by $M_{\mathrm{p}}, R_{\mathrm{p}}$ and $\rho_{\mathrm{p}}$, only two of which need to be specified.

Observationally, constraints on these properties are minimal. Giant branch YORP discs have not yet been observed, spin discs might have been generated in just one known system (Vanderbosch et al. 2020), and tidal discs just have an assumed lower radius grain limit of about $1.5 \mu \mathrm{m}$ (Xu et al. 2018a).

In fact, the spherical assumption, which would have negated the YORP effect, itself is probably inappropriate, given e.g. the complexity of the transiting signatures seen in the WD $1145+017$ white dwarf planetary system (Vanderburg et al. 2015) from, for example, Gänsicke et al. (2016), Garv et al. (2017), Izquierdo et al. (2018), Rappaport et al. (2018) and Xu et al. (2019). The size of the one large planetesimal orbiting within the disc around SDSS J1228+1040 (Manser et al. 2019) is uncertain by a few orders of magnitude (with a radius range of about 4-600 km), and likely represents a core fragment of density $\rho_{\mathrm{p}} \gtrsim 7.7 \mathrm{~g} / \mathrm{cm}^{3}$ with non-zero internal strength.

Theoretical models, however, have established other constraints. The highest possible mass of the progenitor of the planetesimals orbiting WD $1145+017$ is thought to be about 10 per cent the mass of Ceres, such that $M_{\mathrm{p}} \lesssim 10^{20} \mathrm{~kg}$ (Rappaport et al. 2016; Veras et al. 2017a; Gurri et al. 2017). The density of this progenitor, however, is not necessarily uniform (Duvvuri et al. 2020), leaving $\rho_{\mathrm{p}}$ unconstrained. Indeed, a disc composed of solely iron-rich core fragments is not unreasonable (Manser et al. 2019), just as is a disc composed of only porous rubble piles.

We can also look for theoretical constraints in studies of disc formation, whether it be from tidal disruption (Graham et al. 1990; Jura 2003; Debes et al. 2012; Veras et al. 2014a; Malamud \& Perets 2020a.b) or rotational disruption (Makarov \& Veras 2019; Veras et al. 2020a). Malamud \& Perets (2020b) illustrated that tidal disruption can produce planetesimals ranging in size from their numerical resolution limit of a few $\mathrm{km}$ up to hundreds of $\mathrm{km}$. The size distribution of fragments resulting from rotational disruption remains unclear, but would be a strong function of the number of fissions (Scheeres 2018; Veras \& Scheeres 2020).

The size distribution in YORP discs depends on the number of YORP-induced fissions, as well as the internal strength, density and shapes of the planetesimals. Veras \& Scheeres (2020) modelled planetesimals in the size 


\section{White dwarf debris discs}

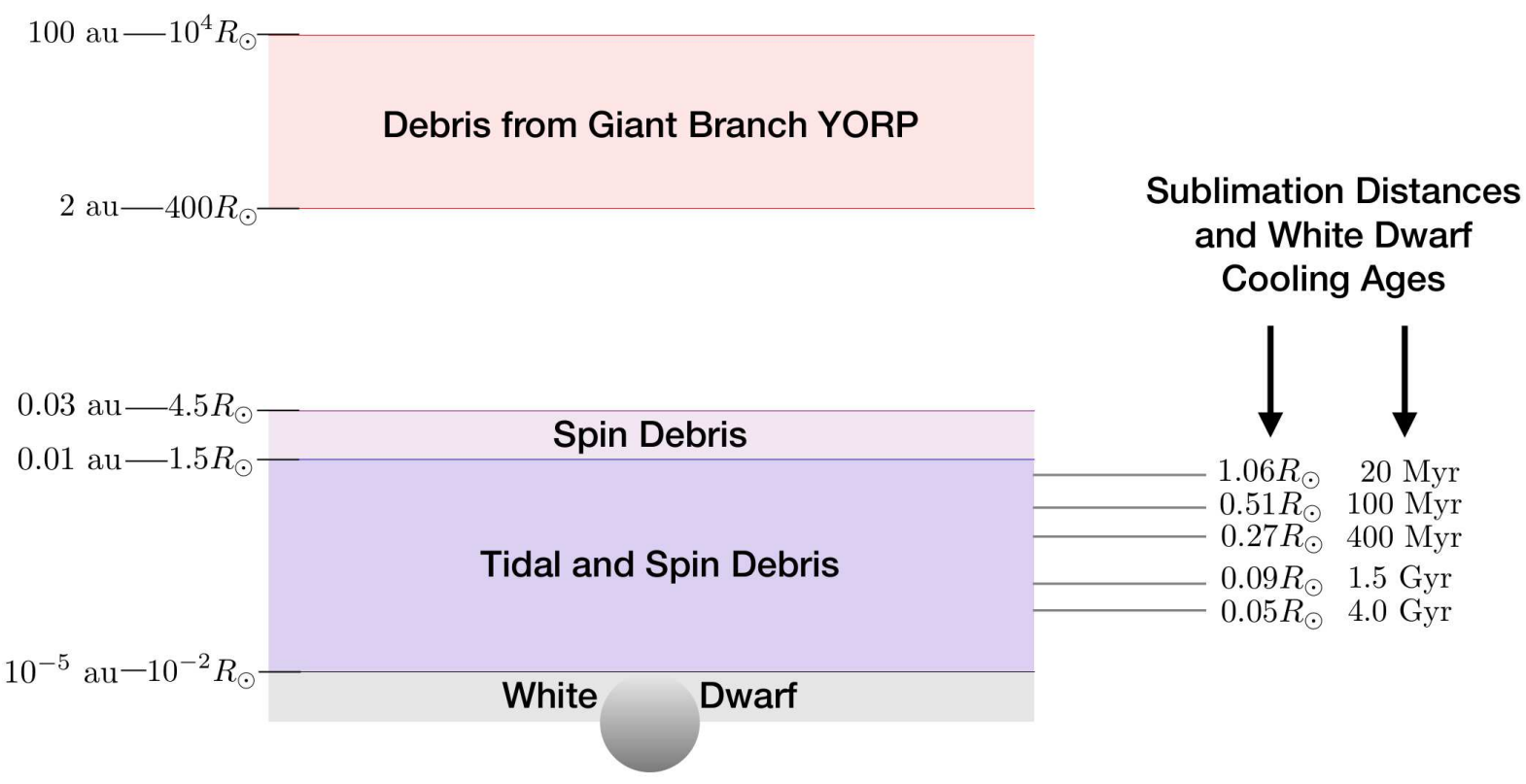

Figure 1. A cartoon with logarithmic vertical spacing which illustrates the predominant types and spatial extents of debris discs orbiting white dwarfs. Not included are exo-Oort clouds, which would be largely depleted, nor pockets of debris which could exist in stable resonant configurations with surviving major planets. Exo-asteroids which avoid engulfment into the star during the giant branch phases would likely have arrived around the white dwarf at a separation of at least 2 au and in a fragmented state from the radiative YORP effect. Surviving fragments which are then perturbed towards the white dwarf (by surviving major planets) could be (further) broken up due to radiation-less spin disruption or tidal disruption; $1.5 R_{\odot}$ represents the approximate maximum tidal disruption separation for solid bodies. Given on the right are fiducial sublimation distances as a rough function of white dwarf cooling age; they indicate where a tidal debris disc can no longer be considered to be gas-free.

range of $1 \mathrm{~m}$ to $10 \mathrm{~km}$, but were non-committal about the size of the smallest monolithic constituents of progenitor asteroids. A wider range of planetesimals may comprise discs formed from the fragments of collisions amongst major planets. However, such collisions are expected to be rare (most gravitational instability events induce escape; see Appendix A of Veras et al. 2016a) compared to YORP processes, which would be near-ubiquitous.

Overall, we characterize the planetesimals by their radius $R_{\mathrm{p}}$ and density $\rho_{p}$, where $M_{\mathrm{p}}$ is then obtained trivially. We adopt the ranges $R_{\mathrm{p}}=10^{-5}-10^{2} \mathrm{~km}$ and $\rho_{\mathrm{p}}=1-8$ $\mathrm{g} / \mathrm{cm}^{3}$.

\subsection{The disc: $r_{\mathrm{i}}, r_{\mathrm{o}}, M_{\mathrm{disc}}, \sigma_{e}, \sigma_{i}$}

As for the disc, we parametrize it with five variables: inner radius $r_{\mathrm{i}}$, outer radius $r_{\mathrm{o}}$, total mass $M_{\mathrm{disc}}$, and the root-mean-squared eccentricity $\sigma_{e}$ and inclination $\sigma_{i}$ of the ensemble of its constituents.

We assume that the disc is a gas-free circular annulus. For tidal and perhaps spin discs, this structure may also be referred to as a "ring" because the spatial extent of the detected dust is usually comparable in range to that of some of Saturn's rings. However, the simulations of Malamud \& Perets (2020b) show that in fact tidal disruption can spread planetesimals over a much wider range, whereas the transiting signatures around ZTF J0139+5245 (Vanderbosch et al. 2020) suggest the presence of a much narrower ring of material. Although this particular ring is likely to be extremely eccentric (with $e \approx 0.97$ ), most dusty disc/ring structures are observed to be near circular (Rocchetto et al. 2015; Farihi 2016). In reality, the situation is more complicated, and the eccentricity may be a function of semimajor axis (Manser et al. 2016a), with intensity patterns in at least the gas surrounding SDSS J1228+1040 harbouring eccentricities of potentially several tenths (Manser et al. 2019).

We confine our annuli to the three classes of discs defined in Fig. 1, which we henceforth refer to as "YORP discs", "spin discs", and "tidal discs". We now provide additional detail about their constraints.

\subsubsection{The inner radius $r_{\mathrm{i}}$}

For YORP discs, the minimum disc edge is set by the engulfment distance along the giant branch phases, which is a few au (Kunitomo et al. 2011; Mustill \& Villaver 2012; Adams \& Bloch 2013; Nordhaus \& Spiegel 2013; Villaver et al. 2014; Madappatt et al. 2016; Staff et al. 2016; Gallet et al. 2017; Rao et al. 2018; Sun et al. 2018; Ronco et al. 2020). Further, because YORP breakup may readily occur at several tens of au, we set the maximum 
value of $r_{\mathrm{i}}$ at $30 \mathrm{au}$. Hence for these discs we set $r_{\mathrm{i}}=2-30$ au.

For spin and tidal discs, given our gas-free assumption, the value of $r_{\mathrm{i}}$ is set by the sublimation distance from the white dwarf as a function of $t_{\mathrm{cool}}$, and hence $L_{\star}$ and $T_{\star} ;$ Fig. 1 provides some approximate values of the sublimation distance as a function of $t_{\mathrm{cool}}$. We denote $r_{\mathrm{sub}}$ as an idealized distance beyond which no material is sublimated; in fact, as indicated by Jura (2008) and Section 6.1.1 of Veras (2016a), the sublimation rate of a planetesimal is better characterized as a continuous function of separation from the star. Nevertheless, for our purposes, we adopt the useful basic prescription of Rafikov (2011b)

$r_{\mathrm{sub}}=\frac{1}{2} R_{\star}\left(\frac{T_{\star}}{T_{\mathrm{sub}}}\right)^{2}$

where $T_{\text {sub }}$ is the sublimation temperature of a particular substance. Table 1 of Rafikov \& Garmilla (2012) show that $T_{\text {sub }}=1600,2000,2100,2300,2300,2600 \mathrm{~K}$ for, respectively, iron, CAIs, olivine, $\mathrm{SiC}, \mathrm{Al}_{2} \mathrm{O}_{3}$ and graphite.

The range of $r_{\text {sub }}$ is extensive. The minimum possible value of $r_{\text {sub }}$ is obtained by considering the minimum $T_{\star}$ of about $4000 \mathrm{~K}$, the maximum $T_{\text {sub }}$ corresponding to graphite, and a value of $R_{\star}$ corresponding to $M_{\star}=0.8 M_{\odot}$. Those yield $\min \left(r_{\text {sub }}\right)=1.19 R_{\star}$. In contrast, for the youngest white $\operatorname{dwarfs}, \max \left(r_{\text {sub }}\right)>1.0 R_{\odot}$.

Overall, for tidal and spin discs, we set $r_{\mathrm{i}}=0.4 R_{\star}-$ $1.0 R_{\odot}$.

\subsubsection{The outer radius $r_{\mathrm{o}}$}

Observations are currently limited to tidal disc: 3 , where $r_{\mathrm{o}}=1.0-1.2 R_{\odot} \approx 4.6-5.5 \times 10^{-3}$ au (e.g. Gänsicke et al. 2006). This distance is commonly thought to coincide with the Roche, or tidal disruption distance $r_{\text {Roche, although }}$ $r_{\text {Roche }}$ can actually vary considerably depending on the material properties of the planetesimal. For solid bodies, Veras et al. (2017a) illustrated that $r_{\text {Roche }} \approx 0.5-1.5 R_{\odot}$. Hence, for tidal discs, we adopt $\max \left(r_{\mathrm{o}}\right)=1.3 R_{\odot}$.

The extent of spin discs remains unclear. Makarov \& Veras (2019) demonstrated the concept of YORP-less spin-up to destruction due solely to close passages with a white dwarf, and Veras et al. (2020a) applied the idea to the ZTF J0139+5245 system, where the semimajor axis of the debris is just 0.42 au (Vanderbosch et al. 2020). Veras et al. (2020a) found that rotational fission could occur at separations up to $3 R_{\text {Roche }}$ across the progenitor density range of $1-8 \mathrm{~g} / \mathrm{cm}^{3}$. We adopt $\max \left(r_{\mathrm{o}}\right)=4.5 R_{\odot}$ here for these discs, but acknowledge that spin discs may extend further pending future parameter space explorations.

Regarding YORP discs, observational searches for rocky debris beyond $\approx 1.0 R_{\odot}$ have been carried out by $\mathrm{Xu}$ et al. (2013) and Farihi et al. (2014) for the white dwarf planetary systems GD 362 and G29-38, respectively. These searches extended to tens of au, with a correspondingly decreasing ability to constrain the mass. For example, around GD 362 , at 5 au the dust mass was constrained to be no larger

3 However, Su et al. (2007) provided tantalizing hints of a YORP disc extending many tens of au to over $10^{2}$ au. than about $10^{22} \mathrm{~kg}$ (more massive than Ceres). The constraints were better for G29-38; at $11 \mathrm{au}$, the dust mass was constrained to be no larger than about $3 \times 10^{21} \mathrm{~kg}$. Hence, massive planetesimal discs out to tens of au may exist but remain undetected. For YORP discs, we hence adopt $\max \left(r_{\mathrm{o}}\right)=100 \mathrm{au}$ but acknowledge that they could extend further (Veras et al. 2019a).

All of the maximum values of $r_{\mathrm{o}}$ are subject to a condition on the width of the annulus; the analytical formalism of Heng \& Tremaine (2010) relies on a disc which is neither too extended nor narrow. To quantify the restriction on the disc's extent, we use the $f_{m}$ parameter from Heng \& Tremaine (2010)

$f_{m} \equiv \frac{4\left(r_{\mathrm{o}}-r_{\mathrm{i}}\right)}{r_{\mathrm{o}}+r_{\mathrm{i}}}$.

In order for the formalism to hold, $f_{m}$ should be of order unity, meaning $r_{\mathrm{o}} / r_{\mathrm{i}} \sim 5 / 3$. We will adopt the range $1 / 2<$ $f_{m}<3$, which corresponds to $9 / 7 \leqslant r_{\mathrm{o}} / r_{\mathrm{i}} \leqslant 7$.

\subsubsection{The disc mass $M_{\mathrm{disc}}$}

Planetary debris discs orbiting white dwarfs have masses which are poorly constrained observationally. YORP discs have not yet been observed. For tidal systems with transiting debris, the amount of dust may be estimated by assuming that a homogeneous rectangular or cylindrical dust cloud creates the transits (Gänsicke et al. 2016; Xu et al. 2018a; Vanderbosch et al. 2020; Veras et al. 2020a). For both WD $1145+017$ and ZTF J0139+5245, this mass was estimated to be about $10^{14} \mathrm{~kg}$. This value, however, is several orders of magnitude smaller than the disc mass that would be needed to circularize planetesimals in the WD $1145+017$ disc (O'Connor \& Lai 2020).

Alternatively, if nearly every metal-polluted white dwarf harbours an orbiting debris disc which acts as a conduit to the accretion, then we can look to the accumulated mass over the last Myr or so in helium-rich (DBZ spectral type) white dwarf convection zones. This accumulated mass in these stars is $10^{16}-10^{22} \mathrm{~kg}$ (Farihi et al. 2010; Girven et al. 2012; Xu \& Jura 2012; Veras 2016a).

From a theoretical perspective, the lower limit on the disc's mass and surface density can be arbitrarily small. The upper limit is set by the extent of the surviving planetary material from the giant branch phases. One terrestrial planet (of mass $10^{24} \mathrm{~kg}$ ) could easily be kicked towards the white dwarf due to a larger planet Veras et al. 2016a) or a binary stellar companion (Bonsor \& Veras 2015; Hamers \& Portegies Zwart 2016; Petrovich \& Muñoz 2017; Stephan et al. 2017, 2018; Veras et al. 2017b), disrupt and form a disc; such discs are even massive enough to trigger second-generation planetesimal formation (Schleicher \& Dreizler 2014; Völschow et al. 2014; van Lieshout et al. 2018). However, major planets are not nearly as frequent as, say, $10^{22} \mathrm{~kg}$ asteroids within a belt (Bonsor et al. 2011; Debes et al. 2012; Frewen \& Hansen 2014; Antoniadou \& Veras 2016, 2019; Mustill et al. 2018; Smallwood et al. 2018), or as frequent as moons around one of the surviving planets (Pavne et al. 2016, 2017).

The progenitor minor planets in YORP discs could result from a giant impact and subsequent breakup of a terrestrial planet $\left(\sim 10^{24} \mathrm{~kg}\right)$. Alternatively, the disc could be 
similar in mass to the solar system's Main Belt $\left(\sim 10^{21} \mathrm{~kg}\right)$, or a much less massive disc.

Overall then, we establish a disc mass range encompassing all of the above estimates: $M_{\text {disc }}=10^{12}-10^{24} \mathrm{~kg}$, or $1.7 \times 10^{-13}-1.7 \times 10^{-1} M_{\oplus}$.

\subsubsection{The dispersions $\sigma_{e}$ and $\sigma_{i}$}

Several white dwarf dusty and gaseous planetary discs showcase dynamical activity. However, a procedure for mapping this activity into a root mean square eccentricity $\left(\sigma_{e}\right)$ and inclination $\left(\sigma_{i}\right)$ of the planetesimals (and then back to their progenitors) is not obvious. Nevertheless, we must set these variables as inputs, and have deliberately generalized the treatment of Heng \& Tremaine (2010) and Heng \& Malik (2013) So that $\sigma_{e}$ and $\sigma_{i}$ are not set at a fixed ratio.

For systems with infrared excesses, the dynamical activity arises from changes in this flux over time (Xu \& Jura 2014; Farihi et al. 2018; Xu et al. 2018b; Swan et al. 2019a; Wang et al. 2019; Rogers et al. 2020). Although the more sudden changes could arise from a recent impact event (from perhaps an asteroid colliding with the extant disc; Wang et al. 2019), the flux changes more likely result from interactions amongst the dust itself and potentially with the gas (when it exists). Variability within the gaseous components (Wilson et al. 2014; Manser et al. 2016b; Redfield et al. 2017; Cauley et al. 2018; Dennihy et al. 2018) takes on a different form, but is not as directly related to our choices for $\sigma_{e}$ and $\sigma_{i}$ for solid debris.

All this activity arises from observed tidal discs (within $\left.1.5 R_{\odot}\right)$, where eccentricity and inclination changes are limited. As a dynamical comparison, these limitations do not exist in the aforementioned giant branch YORP discs. The planetesimals in those discs can be travelling on orbits which encompass the entire range of eccentricity and inclination. The reason is because prior to break-up, the asteroid's orbit was likely altered through a "supercharged" radiative Yarkovsky effect, thousands of times more powerful than that in the solar system (Veras et al. 2015a, 2019a).

Therefore, we do not uniformly adopt the assumption of Heng \& Tremaine (2010) that $\sigma_{i}=(1 / 2) \sigma_{e}$. This longstanding relation appeared to originate in the protoplanetary disc literature from both simulations (Cazenave et al. 1982) and analytical formulations (Hornung et al. 1985). However, the formation channels of those discs are different than all of the ones considered in this paper, and those discs were often assumed near-coplanar and near-circular.

Hence, in our computations, when $\sigma_{e} \ll 0.1$, we will often assume $\sigma_{i}=(1 / 2) \sigma_{e}$ as a default choice, but not always. In our analytical treatment, we leave $\sigma_{e}$ and $\sigma_{i}$ as free parameters which obey a Rayleigh distribution. $\sigma_{e}$ is naturally constrained by the boundaries of the disc, such that

$0<\sigma_{e} \ll \frac{r_{\mathrm{o}}-r_{\mathrm{i}}}{r_{\mathrm{o}}+r_{\mathrm{i}}}$.

We assume that $\sigma_{i}$ is equivalently restricted such that

$0<\sigma_{i} \ll \frac{r_{\mathrm{o}}-r_{\mathrm{i}}}{r_{\mathrm{o}}+r_{\mathrm{i}}}$.

This restriction on the inclination is necessitated by the formalism but may not be reflective of the high and retrograde inclinations which are easily achieved in YORP discs (Veras et al. 2015a, 2019a).

\subsection{The end state of discs}

Eventually the spin and tidal debris are broken up into dust and gas and accrete onto the white dwarf. So can the accretion process and rates help inform our initial conditions?

The interaction between this gas and the dust is nontrivial and complicates the interpretation of the accretion rate (Metzger et al. 2012). Further, if the dust is replenished or altered (O'Connor \& Lai 2020) regularly from incoming debris, then the equilibrium condition of the dust can alternate between high and low states, with or without the presence of gas (Kenvon \& Bromlev 2017a b). Further, incoming asteroids may bypass the spin or tidal discs entirely and collide with the photosphere of the white dwarf (Brown et al. 2017). If the white dwarf is polluted simultaneously from the gas and from "direct hits", then the accretion rate may contain both stochastic and steady elements (Wvatt et al. 2014; Turner \& Wvatt 2020). None of these complications provide sufficiently constrained predictions for us to alter our parameter choices.

\subsection{Parameter range summary}

We summarize the ranges for our initial parameters here, and note that we will perform a systematic march through the phase space. Unlike Heng \& Malik (2013), we do not pursue a Monte Carlo approach.

(i) For all disc types, $M_{\star}=0.4-0.8 M_{\odot}$.

(ii) For all disc types, $R_{\mathrm{p}}=10^{-5}-10^{2} \mathrm{~km}$.

(iii) For all disc types, $\rho_{\mathrm{p}}=1-8 \mathrm{~g} / \mathrm{cm}^{3}$.

(iv) For YORP discs, $r_{\mathrm{i}}=2-30 \mathrm{au}$. For tidal and spin $\operatorname{discs} r_{\mathrm{i}}=0.4 R_{\odot}-1.5 R_{\odot}$.

(v) Subject to the restriction $9 / 7 \leqslant r_{\mathrm{o}} / r_{\mathrm{i}} \leqslant 7$ : for YORP discs, $\max \left(r_{\mathrm{o}}\right)=100 \mathrm{au}$; for spin discs, $\max \left(r_{\mathrm{o}}\right)=4.5 R_{\odot}$; and for tidal discs, $\max \left(r_{\mathrm{o}}\right)=1.3 R_{\odot}$.

(vi) For all disc types, $M_{\text {disc }}=10^{12}-10^{24} \mathrm{~kg}$.

(vii) For all types of discs,

$0<\sigma_{e} \ll \frac{r_{\mathrm{o}}-r_{\mathrm{i}}}{r_{\mathrm{o}}+r_{\mathrm{i}}}$.

(viii) For all types of discs,

$0<\sigma_{i} \ll \frac{r_{\mathrm{o}}-r_{\mathrm{i}}}{r_{\mathrm{o}}+r_{\mathrm{i}}}$.

\section{DISC LIFETIME}

The primary goal of this paper is to compute the maximum lifetime, $t_{\mathrm{disc}}$, of three types of debris discs which orbit white dwarfs by using the analytical formalism of Heng \& Tremaine (2010) and Heng \& Malik (2013), thereby avoiding numerical integrations. As a first step, we define a disc as a structure which contains at least 10 planetesimals, such that the following condition must be satisfied

$R_{\mathrm{p}} \leqslant\left(\frac{3 M_{\mathrm{disc}}}{20 \pi \rho_{\mathrm{p}}}\right)^{1 / 3}$. 


\subsection{Initial auxiliary quantities}

In order to compute $t_{\text {disc }}$, we first compute a series of relevant physical quantities. The first is the effective semimajor axis $a$ of the disc

$a \equiv \frac{1}{2}\left(r_{\mathrm{i}}+r_{\mathrm{o}}\right)$.

Then we can define the Hill radius, $R_{\text {Hill }}$, of an individual planetesimal. The literature contains several expressions for $R_{\mathrm{Hill}}$, some of which include an eccentricity dependence (e.g. Pearce \& Wyatt 2014) and others which refer to the mutual Hill radius between two objects (e.g. Chambers et al. 1996; Stewart \& Ida 2000). For our purposes, we follow Heng \& Tremaine (2010) and define

$R_{\text {Hill }} \equiv a\left(\frac{M_{\mathrm{p}}}{3 M_{\star}}\right)^{\frac{1}{3}}$.

Because the same value of $a$ would be applied to each planetesimal rather than the instantaneous separation of that particular planetesimal, the formalism becomes less applicable as the disc's extent $\left(r_{\mathrm{o}}-r_{\mathrm{i}}\right)$ is increased.

The height $h$ of the disc is given by

$h=\frac{a \sigma_{i}}{\sqrt{2}}$

and the surface mass density $\Sigma$ is

$\Sigma=\frac{M_{\mathrm{disc}}}{\pi f_{m} a^{2}}$

from which follows the total mass density in the midplane

$\rho_{0}=\frac{\Sigma}{\sqrt{\pi} a \sigma_{i}}$

as well as the number density in the midplane

$n_{0}=\frac{\Sigma}{\sqrt{2 \pi} M_{\mathrm{p}} h}$.

The mean motion of the disc, which is more commonly characterized as the orbital frequency $\Omega$, is

$\Omega=\sqrt{\frac{G M_{\star}}{a^{3}}}$

and the radial velocity dispersion $\sigma_{r}$ is

$\sigma_{r}=\frac{a \sigma_{e} \Omega}{\sqrt{2}}$

With these variables, we can also define the Safronov number $\Theta$, which conveys the effect of gravitational focussing in scattering calculations

$\Theta=\frac{G M_{\mathrm{p}}}{2 \sigma_{r}^{2} R_{\mathrm{p}}}$

and a quantity $\Delta a$ which characterizes the typical radial separation between planetesimals, with

$\Delta a=\frac{M_{\mathrm{p}}}{2 \pi \Sigma a}$.

\subsection{Toomre stability}

With these quantities, we can determine if the disc is gravitationally stable to axisymmetric perturbations, a stability criterion known as Toomre stability (Toomre 1964). The disc is Toomre unstable when either
$M_{\mathrm{p}}<\frac{4 \pi^{3} \Sigma^{2} a^{4}}{M_{\star}} \quad$ and $\quad \sigma_{e}<\frac{\sqrt{2} \pi \Sigma a^{2}}{M_{\star}}$

or when

$$
\begin{array}{r}
M_{\mathrm{p}} \geqslant \frac{4 \pi^{3} \Sigma^{2} a^{4}}{M_{\star}} \quad \text { and } \quad 1>\frac{M_{\mathrm{p}} M_{\star}}{(2 \pi)^{3} \Sigma^{2} a^{4}} \\
\text { and } \quad \sigma_{e}<\sqrt{\frac{M_{\mathrm{p}}}{\pi M_{\star}}\left[1-\frac{M_{\mathrm{p}} M_{\star}}{(2 \pi)^{3} \Sigma^{2} a^{4}}\right]} .
\end{array}
$$

We only compute maximum disc lifetimes for discs which are Toomre stable.

\subsection{Disc classification}

Heng \& Tremaine (2010) made the distinction between "cold", "warm" and "hot" discs, depending on a number of factors. These distinctions crucially determine how $t_{\text {disc }}$ is computed. Cold discs are discs where most of the planetesimal orbits do not cross, a condition which is equivalent to

$\sigma_{e}<\frac{\left|\Delta a-2 R_{\mathrm{p}}\right|}{2 a}$.

In this case, the disc leaves the steady state only after the inherently chaotic nature of the multi-body problem generates a scattering event. We refer to this instability timescale as $t_{\text {chaos }}$, which we estimate with the given parameters in the next subsection.

In contrast, both "warm" and "hot" discs feature collisions. If the collisions do not change the mass distribution, then the disc is "warm". Otherwise, the disc is "hot". Changing the mass distribution would entail any one of the following three events occurring: (i) collisions generating gravitationally bound pairs, (ii) collisions excessively eroding away the planetesimals, or (iii) the disc undergoing excessive viscous spreading.

These three events can all be parametrized. When $\Theta>1$, then collisions can generate gravitationally bound pairs. Collisions can be thought of as gradually chipping away at the planetesimals on a timescale $t_{\text {eros }}$, which we estimate with the given parameters in the next subsection. Viscous spreading can be assumed to occur on a timescale $t_{\mathrm{visc}}$, which we can again estimate. Hence, a warm disc requires, in addition to $\Theta \leqslant 1$, that the collisional timescale $t_{\text {coll }}$ must be shorter than $t_{\text {eros }}, t_{\mathrm{visc}}$, and $t_{\text {disc }}$.

Because $t_{\text {disc }}$ is the variable we seek, we cannot know a priori if $t_{\text {coll }}<t_{\text {disc }}$. Hence, with the given parameters, we can classify discs as "cold", "hot", or "warm or hot". Further, in all three cases, we can place bounds on $t_{\text {disc }}$. A final consideration is that gravitational scattering is not just limited to the "cold" disc case. However, a collisional version of the gravitational scattering timescale is inherently different than $t_{\text {chaos }}$, and is hence denoted as $t_{\text {grav }}$. When $t_{\text {grav }}<t_{\text {coll }}$, then for hot discs $t_{\text {disc }}<t_{\text {grav }}$. All of these conditions are summarized in Fig. 2

\subsection{Timescale definitions}

We now explicitly estimate all of these timescales with the given variables. 


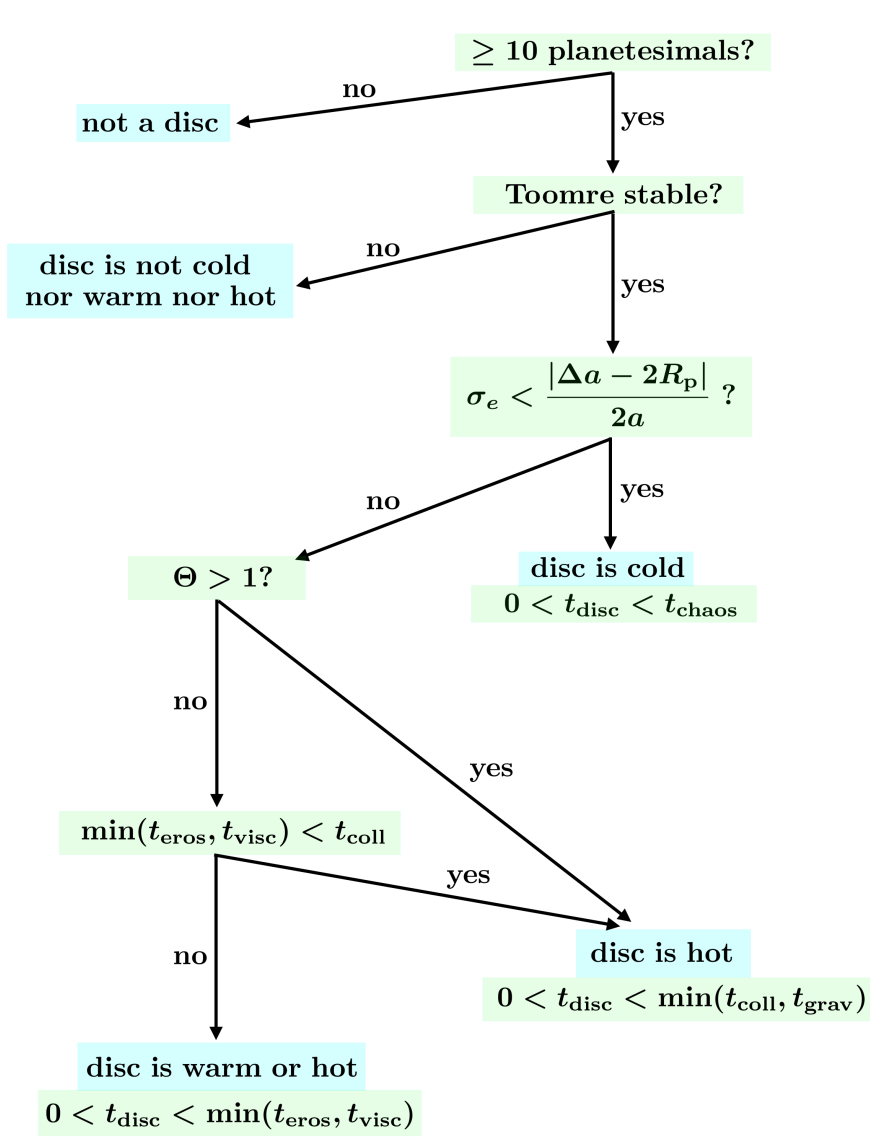

Figure 2. A flowchart describing how debris discs are classified and how disc lifetimes are bounded.

\subsubsection{Collisional timescale $t_{\text {coll }}$}

Heng \& Tremaine (2010) developed an expression for the collision time $t_{\text {coll }}$ by re-formulating the expressions from Greenzweig \& Lissauer (1992) and Dones \& Tremaine (1993) in order to obtain a piecewise function depending on whether the system is in a dispersion-dominated or sheardominated regime. Here, we re-evaluate the expressions in Appendix A of Heng \& Tremaine (2010), but now without assuming a fixed value of $\sigma_{i} / \sigma_{e}$.

There are four cases, the first two being in the dispersion-dominated regime, and the final two in the sheardominated regime.

(i) When $\Theta<1$ and $\sigma_{r} \geqslant \Omega \max \left(R_{\mathrm{p}}, 2^{\frac{1}{3}} R_{\mathrm{Hill}}\right)$, then

$t_{\mathrm{coll}}^{-1}=27.68 \frac{\sigma_{r} \sigma_{i}}{\sigma_{e}} n_{0} R_{\mathrm{p}}^{2}$

(ii) When $\Theta>1$ and $\sigma_{r} \geqslant \Omega \max \left(R_{\mathrm{p}}, 2^{\frac{1}{3}} R_{\text {Hill }}\right)$, then

$t_{\mathrm{coll}}^{-1}=30.49 \frac{\sigma_{i}}{\sigma_{r} \sigma_{e}} n_{0} R_{\mathrm{p}} \Omega^{2} R_{\mathrm{Hill}}^{3}$.

(iii) When

$\Omega \sqrt{2^{\frac{4}{3}} R_{\mathrm{p}} R_{\mathrm{Hill}}} \leqslant \sigma_{r} \leqslant \Omega \max \left(R_{\mathrm{p}}, 2^{\frac{1}{3}} R_{\text {Hill }}\right)$, then

$t_{\mathrm{coll}}^{-1}=56.83 \frac{\sigma_{i}}{\sigma_{e}} n_{0} R_{\mathrm{p}} \Omega R_{\mathrm{Hill}}^{2}$.

(iv) When $\sigma_{r} \leqslant \Omega \sqrt{2^{\frac{4}{3}} R_{\mathrm{p}} R_{\mathrm{Hill}}}$, then $t_{\mathrm{coll}}^{-1}=32.44 \frac{\sigma_{r} \sigma_{i}}{\sigma_{e}} n_{0} R_{\mathrm{p}}^{\frac{1}{2}} R_{\mathrm{Hill}}^{\frac{3}{2}}$.

\subsubsection{Viscous timescale $t_{\mathrm{visc}}$}

The viscous spread of the disc through collisions is

$\left(\frac{\delta a}{a}\right)_{\max }=\sigma_{r} \sqrt{\frac{32 a}{G M_{\star}}}$.

Eq. (42) of Heng \& Tremaine (2010) then illustrated that the viscous timescale is

$t_{\mathrm{visc}}=t_{\mathrm{coll}}\left(\frac{\delta a}{a}\right)_{\max }^{-2}$.

\subsubsection{Erosion timescale $t_{\mathrm{eros}}$}

The erosion timescale $t_{\text {eros }}$ is derived from Section 3.3 of Heng \& Tremaine (2010) as

$t_{\mathrm{eros}}=t_{\mathrm{coll}}\left(\frac{Q_{\mathrm{D}}^{\star}}{\sigma_{r}^{2}}\right)$

where $Q_{D}^{\star}$ represents the scaled disruption energy (or energy per unit mass required to disrupt a planetesimal into fragments).

The value of $Q_{D}^{\star}$ is composition- and size-dependent. Numerous empirical relationships have been developed between this energy and properties of colliding objects; for a recent summary, see Gabriel et al. (2020). Collisions of small bodies are said to be in the strength-dominated regime, whereas collisions of larger bodies are in the gravitydominated regime. Heng \& Malik (2013) provided an expression which includes both regimes:

$Q_{\mathrm{D}}^{\star}=Q_{0}\left[\left(\frac{R_{\mathrm{p}}}{0.2 \mathrm{~km}}\right)^{-0.4}+\left(\frac{R_{\mathrm{p}}}{0.2 \mathrm{~km}}\right)^{1.3}\right]$

where the exponents were chosen to represent a mix of basalt and ice (Benz \& Asphaug 1999), which is a reasonable assumption for debris in white dwarf planetary systems. The constant $Q_{0}$ contains much of the variation that one might expect in $Q_{\mathrm{D}}^{\star}$ over a wide range of $R_{\mathrm{p}}$; we adopt a range of $Q_{0}=10^{4}-10^{7} \mathrm{erg} / \mathrm{g}$, just as in Heng \& Malik (2013).

\subsubsection{Chaos timescale $t_{\text {chaos }}$}

The mutual spacing within which a collection of collisionless bodies would become unstable to gravitational perturbations is a longstanding problem in astrophysics, and one which is often not analytically tractable with more than three bodies.

In planetary science, the discovery of extrasolar planets has inspired many stability applications which are focussed on major planets (Chambers et al. 1996; Chatteriee et al. 2008; Smith \& Lissauer 2009; Funk et al. 2010; Pu \& Wu 2015). For major planet masses, empirical numerical experiments have yielded functional formulae for $t_{\text {chaos }}$ with constant coefficients that are actually a function of the number of the planets, the mass of the planets, and their orbital eccentricities and inclinations. This approach yields only approximate results, and with a lower mass limit $M_{\mathrm{p}} / M_{\star} \sim$ $10^{-10}$, which is unsuitable for the lowest planetesimal masses that we consider here (for $1 \mathrm{~cm}$ pebbles, $M_{\mathrm{p}} / M_{\star} \sim 10^{-33}$ ). 
Therefore, we must find an expression for $t_{\text {chaos }}$ as a function of $M_{\mathrm{p}}$. Equations 3-4 of Zhou et al. (2007) provide such a relation. Written in our variables,

$\log \left(\frac{t_{\text {chaos }}}{1 \text { yr }}\right)=A+U \log \left(\frac{K_{0}}{2.3}\right)$

where

$K_{0} \approx 2^{2 / 3} \frac{a}{R_{\mathrm{Hill}}}\left(\frac{\Delta a}{2 a+\Delta a}\right)$

$A=-2+\sigma_{e}\left(1+\frac{2 a}{\Delta a}\right)-0.27 \log \left(\frac{M_{\mathrm{p}}}{M_{\star}}\right)$,

and

$$
\begin{aligned}
U= & 18.7+1.1 \log \left(\frac{M_{\mathrm{p}}}{M_{\star}}\right) \\
& -\left[16.8+1.2 \log \left(\frac{M_{\mathrm{p}}}{M_{\star}}\right)\right] \sigma_{e}\left(1+\frac{2 a}{\Delta a}\right) .
\end{aligned}
$$

Because these coefficients were derived for systems of 10 planets, one might question their applicability to the pebbles and boulders which we will sometimes consider. However, although these coefficients would change for fewer planets, Funk et al. (2010) indicated that for more than 10 planets, $t_{\text {chaos }}$ becomes independent of the number of planets.

\subsubsection{Gravitational timescale $t_{\text {grav }}$}

In order to compute the gravitational scattering timescale for discs with crossing planetesimal orbits $t_{\text {grav }}$, we use the formalism of Stewart \& Ida (2000). First,

$\left(t_{\mathrm{grav}}\right)^{-1}=\frac{d \ln \left(\sigma_{e}^{2}\right)}{d t}=\frac{1}{\sigma_{e}^{2}} \frac{d \sigma_{e}^{2}}{d t}$.

Then, with Eq. (3.29) of Stewart \& Ida (2000) and with our assumption of equal-mass planetesimals,

$\frac{d \sigma_{e}^{2}}{d t}=\frac{\Omega \Sigma a^{2}}{4 M_{\mathrm{p}}}\left\langle P_{\mathrm{vs}}\right\rangle$

where (their Appendix B)

$\left\langle P_{\mathrm{vs}}\right\rangle=\frac{16 M_{\mathrm{p}}^{2} \beta B}{\pi M_{\star}^{2} \sigma_{e} \sigma_{i}} \int_{0}^{1}\left[\frac{5 \mathcal{K}(\kappa)-\frac{12\left(1-\lambda^{2}\right) \mathcal{E}(\kappa)}{1+3 \lambda^{2}}}{\beta^{2}+\left(1-\beta^{2}\right) \lambda^{2}}\right] d \lambda$.

In Eq. (36), $\beta=\sigma_{i} / \sigma_{e}$, and $\mathcal{K}(\kappa)$ and $\mathcal{E}(\kappa)$ are, respectively, complete elliptic integrals of the first and second kind, with argument

$\kappa=\sqrt{\frac{3}{4}\left(1-\lambda^{2}\right)}$.

The variable $B$ an be expressed through Eqs. (2.17) and (6.6-6.8) of Stewart \& Ida (2000) as

$B=\log \left[\frac{\Lambda^{2}+1}{\Lambda_{\mathrm{c}}^{2}+1}\right]-\frac{1}{\Lambda_{\mathrm{c}}^{2}+1}+\frac{1}{\Lambda^{2}+1}$

with

$\Lambda=\frac{M_{\star}}{M_{\mathrm{p}}}\left(\sigma_{e}^{2}+\sigma_{i}^{2}\right)\left(\sqrt{2} \sigma_{i}+\frac{2^{1 / 3} R_{\mathrm{Hill}}}{a}\right)$

and

$\Lambda_{\mathrm{c}}=\left(\frac{M_{\star}}{M_{\mathrm{p}}}\right)\left(\frac{2 R_{\mathrm{p}}}{a}\right)\left(\sigma_{e}^{2}+\sigma_{i}^{2}\right)$

(C) 2020 RAS, MNRAS 000, 119

$$
\times \sqrt{1+\frac{M_{\mathrm{p}} a}{R_{\mathrm{p}} M_{\star}\left[\sigma_{e}^{2}+\sigma_{i}^{2}+2^{-\frac{1}{3}}\left(\frac{R_{\mathrm{Hill}}}{a}\right)^{2}\right]}} .
$$

\subsection{Radiation drag}

One physical process that may act on shorter timescales than collisions, erosion, scattering or viscous spreading is radiation drag from the white dwarf. For most white dwarfs, such drag is negligible compared to the drag generated during the highly luminous giant branch phases (Bonsor \& Wvatt 2010; Dong et al. 2010; Veras et al. 2015a, 2019a; Zotos \& Veras 2020). However, young white dwarfs are particularly luminous $\left(>10^{-1} L_{\odot}\right)$, albeit briefly (for under $10 \mathrm{Myr}$ ). This high luminosity may be important to consider (Veras et al. 2015b), particularly when the Yarkovsky effect is active for young, hot white dwarfs like WD J0914+1914 (Veras 2020).

We can roughly estimate the timescale for a planetesimal to be dragged through about half of the disc due to radiation. Whether or not the Yarkovsky effect is active can make a significant difference to the radiation drag timescale (of three to four orders-of-magnitude), and depends on the shape, spin and internal density distribution of the planetesimals. Hence, here we provide the limiting maximum drag due to the Yarkovsky effect (Eq. 103 from Veras et al. 2015a), as well as the residual drag - which is the PoyntingRobertson drag (Eq. 111 from Veras et al. 2015a) - when the Yarkovsky effect is turned off.

$$
\begin{aligned}
t_{\text {PRdrag }} & =\left(\frac{r_{\mathrm{o}}-r_{\mathrm{i}}}{2}\right)\left(\frac{d a}{d t}\right)_{\mathrm{PR}}^{-1} \approx \frac{\left(r_{\mathrm{o}}-r_{\mathrm{i}}\right) c^{2} M_{\mathrm{p}} a}{R_{\mathrm{p}}^{2} L_{\star}}, \\
t_{\text {maxYark }} & =\left(\frac{r_{\mathrm{o}}-r_{\mathrm{i}}}{2}\right)\left(\frac{d a}{d t}\right)_{\text {maxYark }}^{-1} \\
& \approx \frac{2\left(r_{\mathrm{o}}-r_{\mathrm{i}}\right) c M_{\mathrm{p}} \sqrt{G M_{\star} a}}{R_{\mathrm{p}}^{2} L_{\star}}
\end{aligned}
$$

In Eqs. (41) and (42), $c$ is the speed of light. For every case studied in this paper, we conservatively compute both $t_{\text {PRdrag }}$ and $t_{\text {maxYark }}$ by assuming a relatively high white dwarf luminosity of $0.1 L_{\odot}$ (corresponding roughly to a cooling age of $10 \mathrm{Myr})$. Hence, if both $t_{\text {maxYark }}<t_{\text {disc }}$ and $t_{\text {maxYark }}<10 \mathrm{Myr}$, then we flag the disc lifetime as possibly being dominated by radiation. Depending on the shape and physical properties of the planetesimal, the actual Yarkovsky timescale may be much longer.

The much weaker Poynting-Robertson drag ensures that $t_{\text {PRdrag }}>t_{\text {maxYark }}$, but is not subject to the same level of uncertainty as Yarkovsky drag (which might never activate, or cancel itself through self-regulation). Hence, if both $t_{\text {maxYark }}<t_{\text {disc }}$ and $t_{\text {PRdrag }}<10 \mathrm{Myr}$, then the disc's evolution is definitely dominated by radiation drag (but only for the high luminosity we sample of $10^{-1} L_{\odot}$ and assuming $\left.t_{\text {disc }}=\max \left(t_{\text {disc }}\right)\right)$. As the results in the next section will show, only a small fraction of the discs that we investigate might be dominated by radiation drag, and only a minority of those are definitely dominated by radiation drag. 


\section{YORP debris discs from 2-3 au}
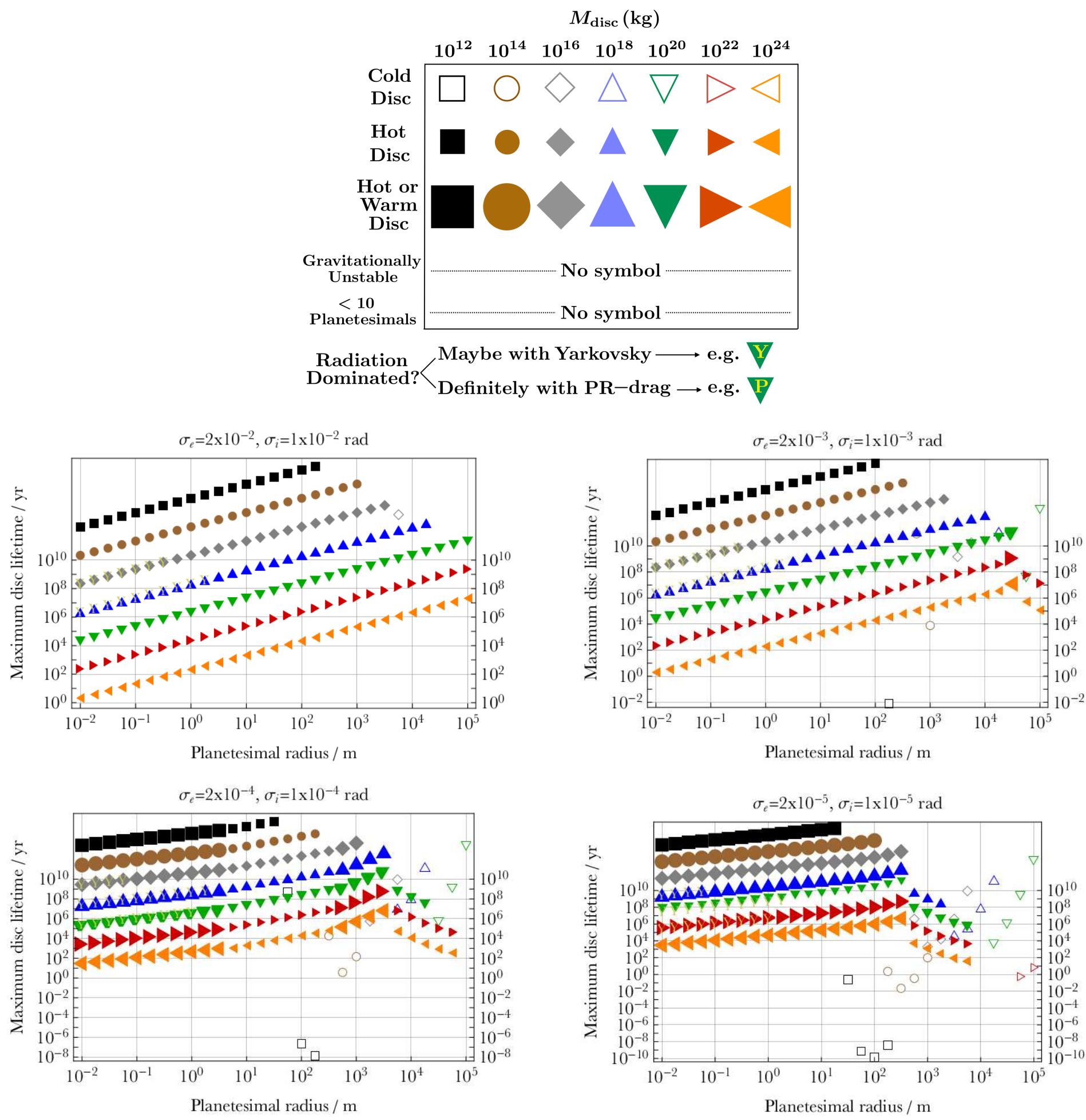

Figure 3. Maximum debris disc lifetimes of narrow and close "Main Belt"-like YORP discs (formed from YORP break up during the giant branch phases). For all plots, $\rho_{\mathrm{p}}=2 \mathrm{~g} / \mathrm{cm}^{3}, M_{\star}=0.6 M_{\odot}, Q_{0}=10^{4} \mathrm{erg} / \mathrm{g}, r_{\mathrm{i}}=2$ au and $r_{\mathrm{o}}=3$ au. The $y$-axis labels do not exceed $10^{10} \mathrm{yr}$ because any symbol above that value indicates a lifetime which exceeds the (approximate) age of the universe. For all plots, discs for which radiation is important ( $\mathrm{Y}$ and $\mathrm{P}$ symbols) always assume a young luminous white dwarf with a constant luminosity of $0.1 L_{\odot}$. The plots illustrate how as the disc becomes flatter and circular, the maximum lifetimes increasingly deviate from clear patterns. Overall, close and narrow YORP discs as massive as about $10^{20} \mathrm{~kg}$ can survive long enough in a steady state to regularly provide a source of intact planetesimals to potentially be perturbed towards the white dwarf. 


\section{YORP debris discs from 30-100 au}
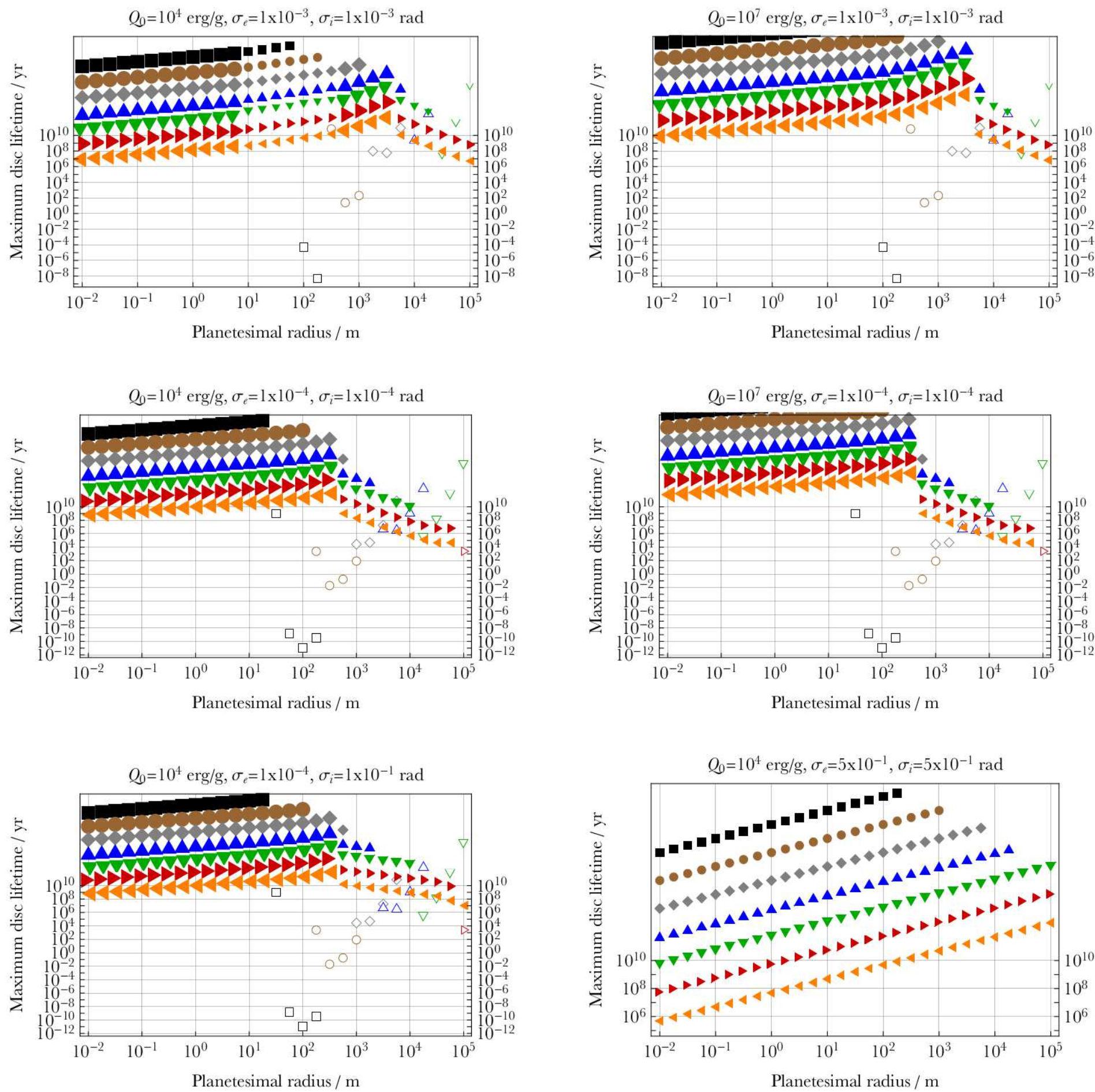

Figure 4. Maximum debris disc lifetimes of broad and distant "Scattered disc"-like YORP discs (formed from YORP break up during the giant branch phase), utilizing the legend in Fig. 3 The plots vary $\sigma_{e}, \sigma_{i}$ and $Q_{0}$ in different combinations, but all assume $\rho_{\mathrm{p}}=2 \mathrm{~g} / \mathrm{cm}^{3}, M_{\star}=0.6 M_{\odot}, r_{\mathrm{i}}=30$ au and $r_{\mathrm{o}}=100 \mathrm{au}$. The plots demonstrate that in all cases, broad YORP discs as massive as $10^{24} \mathrm{~kg}$ live long enough to source intact planetesimals to the white dwarf. 


\section{Spin debris discs from $1.5-4.5 R_{\odot}$}
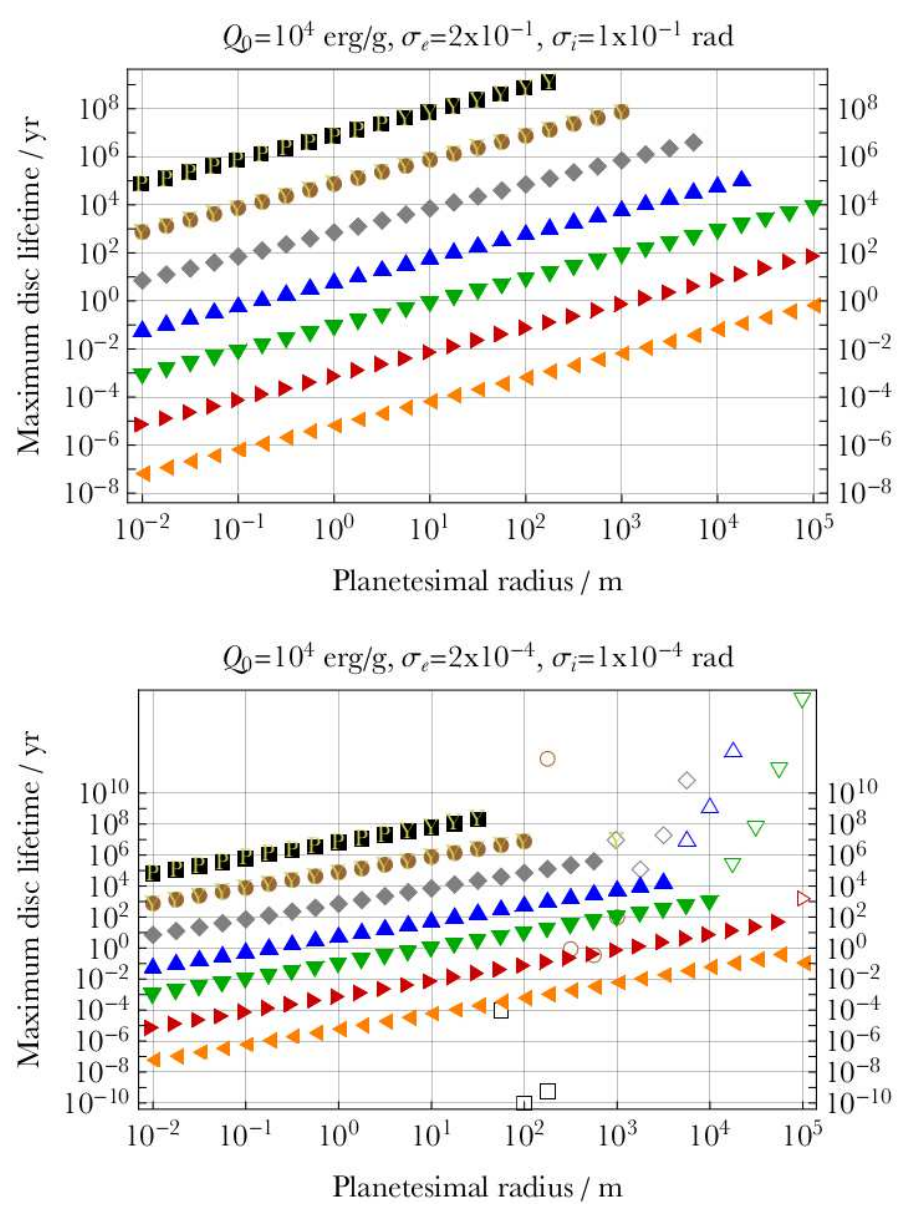
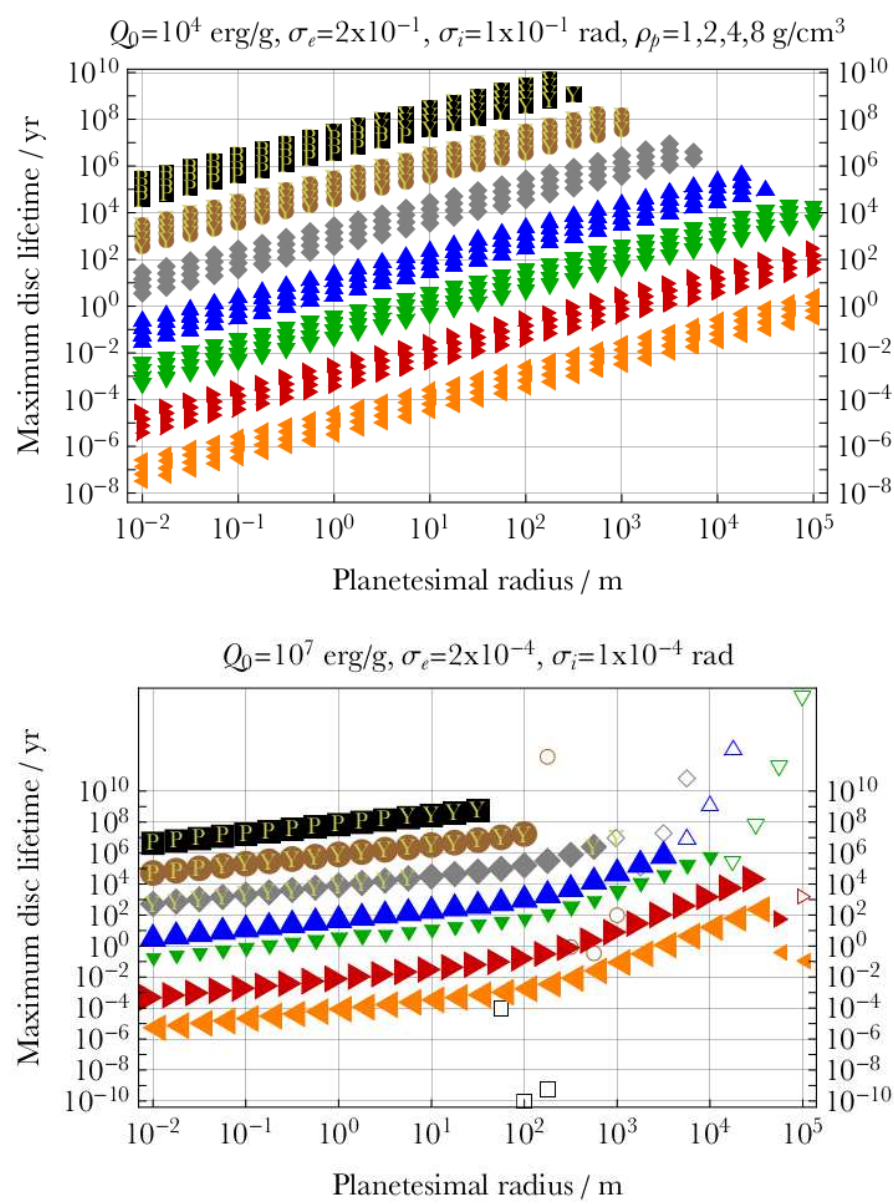

Figure 5. Maximum debris disc lifetimes of spin discs (formed from radiation-less rotational fission during the white dwarf phase), utilizing the legend in Fig. 3 For all plots, $M_{\star}=0.6 M_{\odot}, r_{\mathrm{i}}=1.5 R_{\odot}$ and $r_{\mathrm{o}}=4.5 R_{\odot}$. The upper right plot quantifies the (small) effect of varying $\rho_{\mathrm{p}}$; the bottom symbols correspond to $\rho_{\mathrm{p}}=1 \mathrm{~g} / \mathrm{cm}$ and the top symbols correspond to $\rho_{\mathrm{p}}=8 \mathrm{~g} / \mathrm{cm}$. In all other plots, $\rho_{\mathrm{p}}=2 \mathrm{~g} / \mathrm{cm}$. The plots illustrate that the maximum lifetimes of spin discs can vary from a near instantaneous breakup to about $1 \mathrm{Myr}$ for young, luminous white dwarfs $\left(0.1 L_{\odot}\right)$, depending on both $M_{\text {disc }}$ and $R_{\mathrm{p}}$. Dimmer white dwarfs might admit longer lifetimes when $M_{\text {disc }} \lesssim 10^{16} \mathrm{~kg}$.

\section{RESULTS}

We now compute $\max \left(t_{\text {disc }}\right)$ for ensembles of the three types of debris discs that are illustrated in Fig. 1 YORP-generated discs from the giant branch phases which now orbit white dwarfs (Figs. 314), discs generated from rotational fission during the white dwarf phase (Fig. 5), and discs generated from tidal disruption during the white dwarf phase (Fig. 6. [7). A preliminary analysis of the parameter space revealed that the most useful and illustrative manner in which to present the results is with plots of $\max \left(t_{\mathrm{disc}}\right)$ versus $R_{\mathrm{p}}$, for different curves of $M_{\text {disc }}$.

The legend for all of the plots in Figs. 307 is at the top of Fig. 3. In all plots, maximum lifetimes were computed for the 7 different $M_{\text {disc }}$ values in the legend and for 29 uniformly spaced $R_{\mathrm{p}}$ values from $1 \mathrm{~cm}$ to $100 \mathrm{~km}$. As indicated in the legend, the absence of a symbol indicates that the corresponding combination of $\left(R_{\mathrm{p}}, M_{\mathrm{disc}}\right)$ either does not satisfy the condition for a disc to exist (Eq. 8) or creates a Toomre unstable disc. In order to display all symbols that provide maximum lifetime estimates, the $y$-axes of all plots were extended as necessary, although $10^{10} \mathrm{yr}$ is the highestvalued label that was used to indicate the approximate age of the universe.

\subsection{YORP discs}

We present results for broad (Fig. 3) and narrow (Fig. 4) YORP discs, roughly corresponding to analogues of the solar system's Main Belt and Scattered Disc. Both plots illustrate that the nature of the discs and the maximum disc lifetime does vary depending on how flat and circular the planetesimal orbits are (through $\sigma_{e}$ and $\sigma_{i}$ ). Both the hot discs and the discs labelled as "hot or warm" exhibit a clear correlation between $\max \left(t_{\text {disc }}\right)$ and $R_{\mathrm{p}}$ for sub-m and often sub-km planetesimals. For larger planetesimals, the total number of planetesimals in the disc is fewer, cold discs become a possibility, and hot discs follow a different pattern. 
$\underline{\text { Tidal debris discs from } 0.6-1.2 R_{\odot}}$
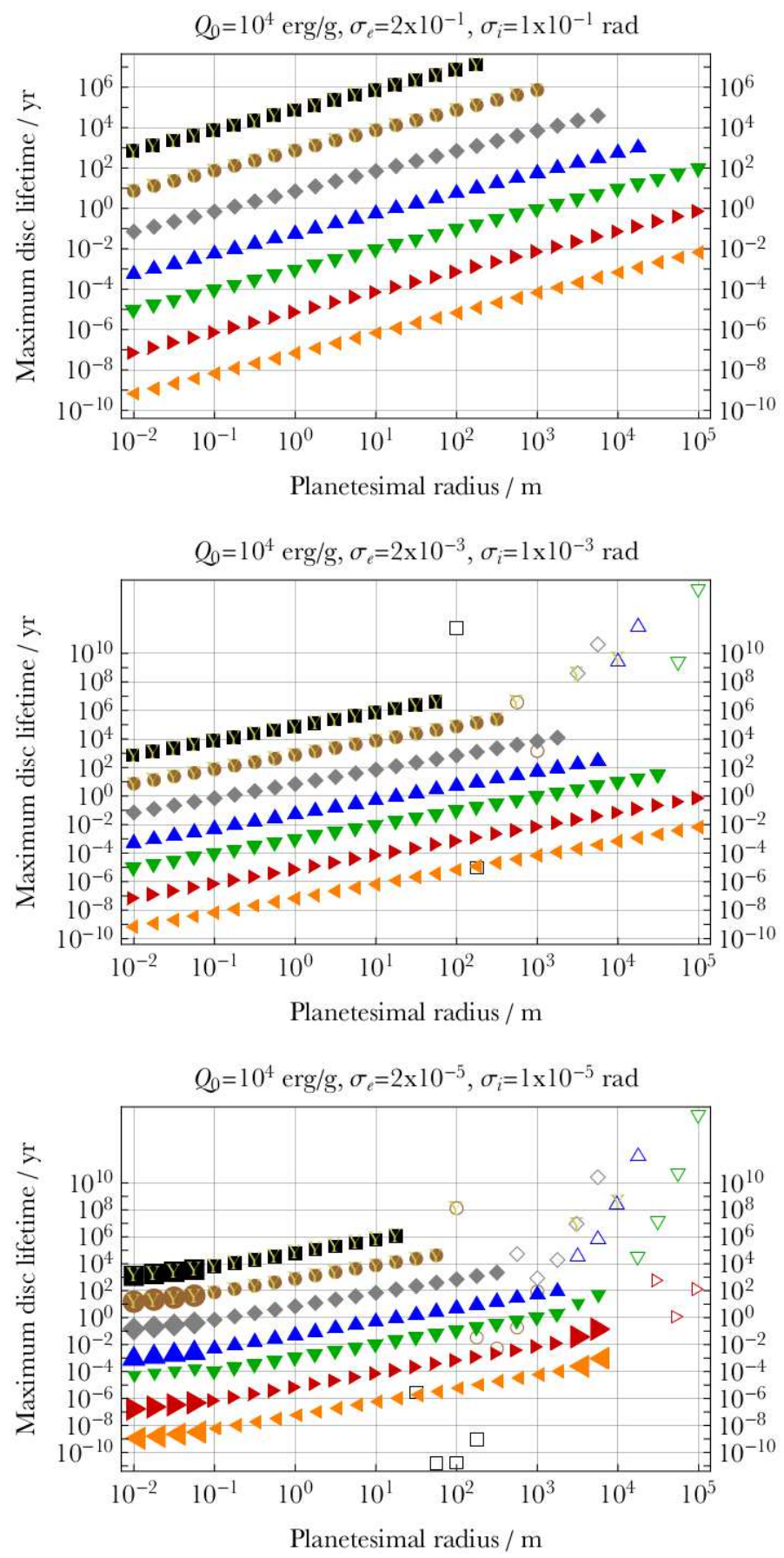
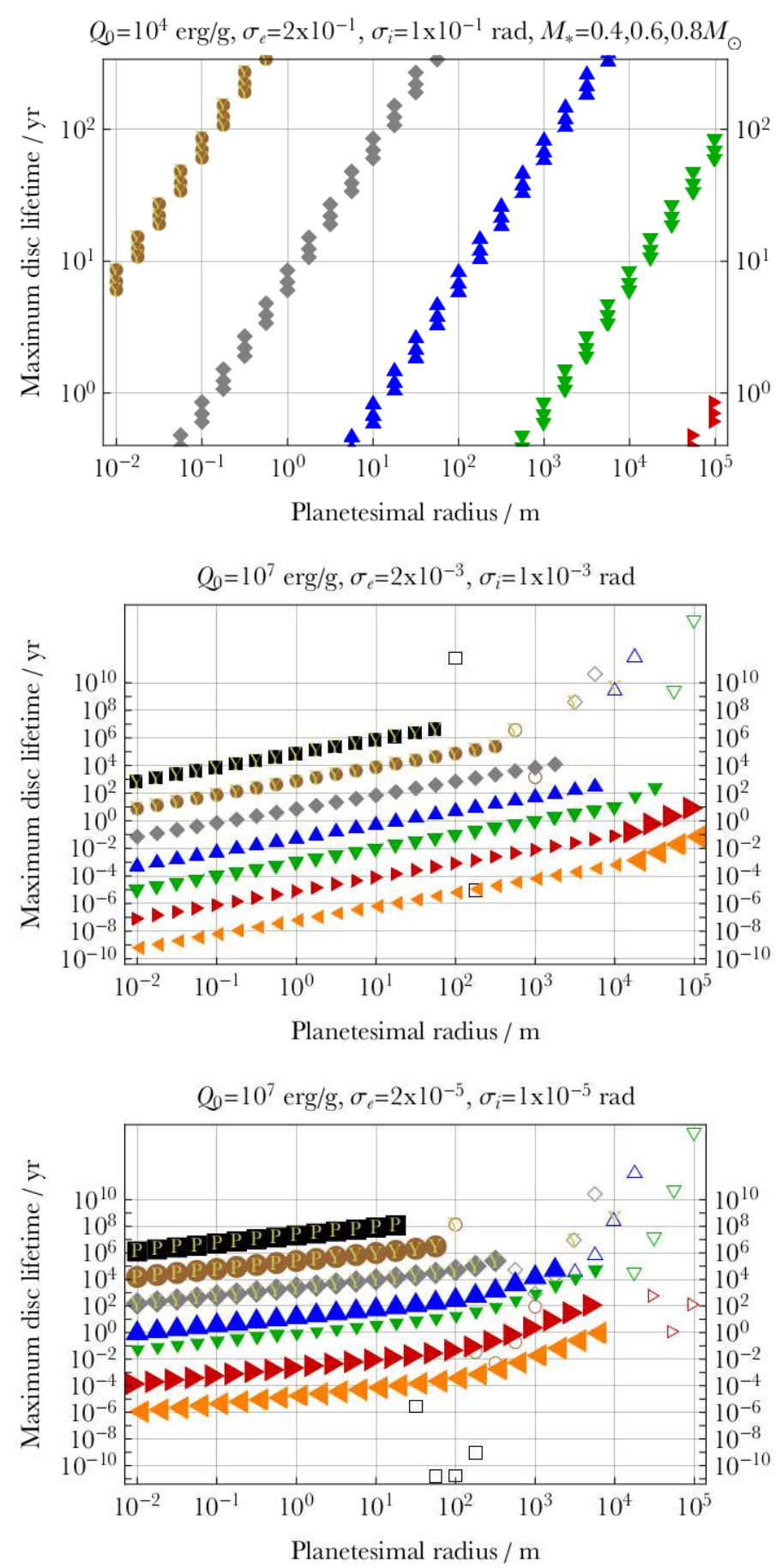

Figure 6. Maximum debris disc lifetimes of broad tidal discs (formed from destruction of bodies which travel into the white dwarf's tidal disruption distance), utilizing the legend in Fig. 3 For all plots, $\rho_{\mathrm{p}}=2 \mathrm{~g} / \mathrm{cm}, r_{\mathrm{i}}=0.6 R_{\odot}$ and $r_{\mathrm{o}}=1.2 R_{\odot}$. The upper right plot quantifies the (very small) effect of varying $M_{\star}$; the top symbols correspond to $M_{\star}=0.4 M_{\odot}$ and the bottom symbols correspond to $M_{\star}=0.8 M_{\odot}$. In all other plots, $M_{\star}=0.6 M_{\odot}$. The plots illustrate that the lifetimes of tidal discs are highly dependent on both $M_{\mathrm{disc}}$ and $R_{\mathrm{p}}$, and may be shorter or longer than observable timescales of years; the maximum disc lifetime is about $10^{4}$ yr, although some cold disc configurations may exist for much longer. 


\section{Tidal debris rings}

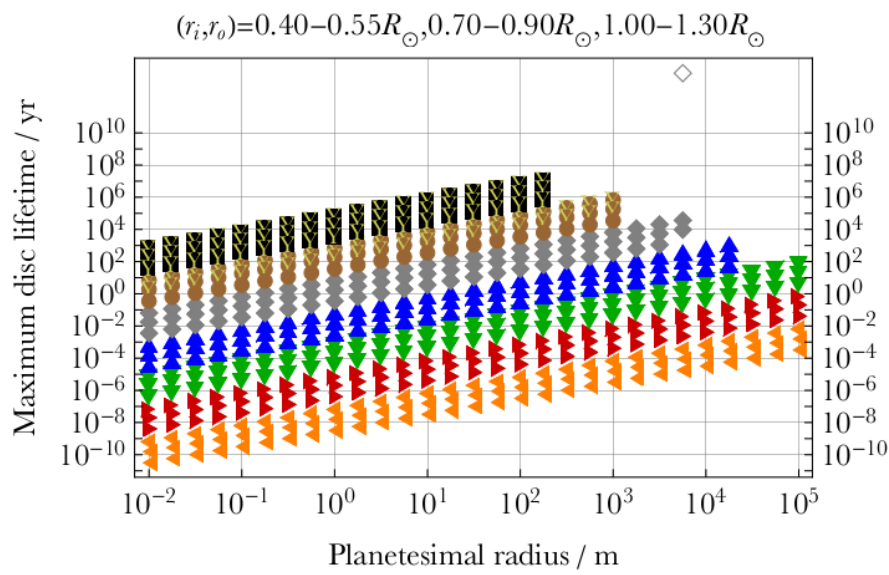

Figure 7. Maximum lifetimes of tidal debris rings (effectively narrow discs formed from destruction of bodies which enter the white dwarf's tidal disruption distance), utilizing the legend in Fig. 3 Here, $M_{\star}=0.6 M_{\odot}, \rho_{\mathrm{p}}=2 \mathrm{~g} / \mathrm{cm}$, and $Q_{0}=10^{4} \mathrm{erg} / \mathrm{g}$. The upper, middle and lower sets of symbols respectively correspond to $\left(r_{\mathrm{i}}-r_{\mathrm{o}}\right)=(1.00-1.30,0.70-0.90,0.40-0.55) R_{\odot}$. The plot illustrates that the maximum lifetimes of tidal debris rings can vary by two orders of magnitude depending on the location of the ring within the tidal zone.

For the narrow 2-3 au YORP discs in Fig. 3 the profiles of the symbol sets become more complex as the disc flattens and becomes more circular. In the most excited case, where $\sigma_{e}=2 \times 10^{-2}=2 \sigma_{i}$, all discs are hot and follow linear trends in logarithmic space for $\max \left(t_{\mathrm{disc}}\right)$ as a function of $R_{\mathrm{p}}$. All discs with $M_{\mathrm{disc}} \leqslant 10^{14} \mathrm{~kg}$ could survive for the age of the universe, as could some with $M_{\text {disc }}=10^{20} \mathrm{~kg}$. Even a few $M_{\text {disc }}=10^{24} \mathrm{~kg}$ discs could survive for up to 10 Myr. More flattened, calm discs may be hot, warm or cold. In no case are the disc lifetimes limited by PoyntingRobertson drag, although $10^{16-20} \mathrm{~kg}$ discs composed of sub$\mathrm{m}$ sized planetesimals may be influenced by the white dwarf luminosity.

The broader 30-100 au YORP discs in Fig. 4 are all too far away to be affected by white dwarf radiation. Their maximum lifetimes are also much higher than in the Main Belt analogue case for sub-km planetesimals and often for larger planetesimals. The six plots in the figure vary $\sigma_{e}, \sigma_{i}$ and $Q_{0}$ to demonstrate the complexity that this variation induces, particularly for the discs composed of the fewest number of planetesimals. The upper two panels are plotted within the same ranges and with the only difference being that $Q_{0}$ varies by three orders of magnitude. The same is true for the middle two panels. These four panels together show that $Q_{0}$ can vary maximum disc lifetimes for "hot or warm" discs only, and by up to three orders of magnitude.

The most excited of these disc sets, with $\sigma_{e}=\sigma_{i}=0.5$, is shown in the bottom right plot. Here, all discs are hot and the only discs which do not survive for at least $10 \mathrm{Gyr}$ are sub-km discs with $M_{\text {disc }} \geqslant 10^{22} \mathrm{~kg}$. If $\sigma_{e} \ll \sigma_{i}$, then the result is shown in the bottom left plot. Comparison with the middle left plot $\left(\sigma_{e}=\sigma_{i}\right)$ showcases only minor differences: only one order of magnitude in $\max \left(t_{\text {disc }}\right)$, and for hot discs only.

\section{$4.2 \quad$ Spin discs}

The spatial scale of spin discs is orders of magnitude smaller than that of YORP discs, but is beyond the tidal radius of the white dwarf. The rough and under-investigated outer boundary of $4.5 R_{\odot}$ reduces the disc lifetime significantly compared to YORP discs, as shown in Fig. 5. Spin discs are also more prone to disruption from white dwarf radiation.

The spatial scales of both spin and tidal discs are comparable to Saturn's rings, which can be near-circular down to the $\sim 10^{-4}$ eccentricity level. However, the formation channel of spin discs could be very different, and the resulting planetesimal orbits may instead feature $\sigma_{e} \gg 10^{-4}$ and $\sigma_{i} \gg 10^{-4}$. Therefore, the upper two plots of Fig. [5 illustrate "excited" cases while the lower two plots instead illustrate "calm" cases. The only difference in the calm case plots is the value of $Q_{0}$ : only for the higher value are warm discs possible. The upper right plot illustrates the effect of altering $\rho_{\mathrm{p}}$; across the entire range of planetesimal density, the maximum disc lifetime varies by less than two orders of magnitude.

All four plots demonstrate that spin discs may reside in a steady state for up to about 1 Myr. However, discs with masses under about $10^{16} \mathrm{~kg}$ could be subject to dominant effects from highly luminous $\left(0.1 L_{\odot}\right)$ white dwarf radiation, and those with $M_{\text {disc }} \approx 10^{12} \mathrm{~kg}$ are often disrupted first by Poynting-Robertson drag.

\subsection{Tidal discs}

Spin debris discs may reside in locations which are up to an order-of-magnitude more distant than tidal debris discs. We explore how such a spatial difference affects $\max \left(t_{\text {disc }}\right)$ for tidal debris discs in Figs. 6017 but first provide a reminder about the assumptions in our model. We are assuming that the disc is gas-free, and hence further away than the sublimation distance, which directly relates to the cooling age (see Fig. 11). Further, for warm and hot discs, any gas produced through collisions is assumed to be negligible. Finally, we assume that the planetesimals themselves, once settled into a disc, do not break apart due to tidal forces.

The overall message conveyed by both Figs. 6 and 7 is that the maximum lifetimes of tidal discs is $\sim 10^{4} \mathrm{yr}$, but may be much shorter, on observable timescales of years. Figure 6 presents broad tidal discs ranging from $0.6-1.2 R_{\odot}$. In the figure, the left panels moving downward feature increasingly flattened and circular tidal discs. The consequence is an increase in the resulting variation of disc types (hot, warm and cold) but a marginal change in the maximum disc lifetimes of the hot and warm discs. As shown in the upper right panel, the dependence of maximum lifetime on $M_{\star}$ is negligible. The other plots in the right panels show discs for which the same initial conditions were adopted as in the left panels, except for a change in the value of $Q_{0}$. Increasing $Q_{0}$ allows for more warm discs to occur, and increases the maximum disc lifetimes by several orders of magnitude.

Figure 7 instead explores the case where tidal disruption produces ring-like instead of disc-like structures (Debes et al. 2012; Veras et al. 2014a; Malamud \& Perets 2020a b). The plot illustrates the variation in the maximum debris disc lifetime due to different placements of narrow discs within $1.3 R_{\odot}$ of the star. This location can vary the 
disc lifetimes by two orders of magnitude (the further away the ring, the higher the maximum lifetime). The innermost regions are only plausible in the first place for the oldest white dwarfs, where the disc avoids sublimation (see Fig. 11).

\section{DISCUSSION}

\subsection{Implications of results}

The implications of our results vary depending on which of the three types of discs is the focus of discussion, although the origin and evolution of all three discs are connected. YORP discs represent an important, if not the primary, source of mass which eventually is perturbed towards and pollutes the white dwarf. Whether or not this mass is already ground down into dust before the perturbation can help determine the rarity of objects like those orbiting WD $1145+017$ and ZTF J0139+5245. The mass of the progenitor of the debris orbiting WD $1145+017$ is thought to contain about $10^{20} \mathrm{~kg}$ of mass Rappaport et al. 2016; Veras et al. 2017a; Gurri et al. 2017) whereas the mass of the progenitor in the ZTF J0139+5245 system is not as well constrained.

Figures 3.4illustrate definitively that YORP discs are a viable reservoir which may supply planetesimals to the white dwarf for cooling ages exceeding 10 Gyr. This result is independent of planetesimal size, which the plots demonstrate could span seven orders of magnitude from $1 \mathrm{~cm}$ to $100 \mathrm{~km}$. Although both plots illustrate unlikely cases where the discs may be cold, the majority are most likely hot. Hot discs are common when $\sigma_{e}, \sigma_{i}>10^{-3}$, and we expect YORP discs to form in dynamically excited states.

The reason is because the giant branch Yarkovsky effect acts concurrently on progenitor asteroids and will have probably already significantly altered their orbital eccentricity and inclination (Veras et al. 2015a, 2019a) before YORP breakup. The typical fragmentation sizes for YORP breakup are unknown, and this process around giant branch stars has yet to be modelled in detail. Veras \& Scheeres (2020) did, however, establish that multiple fission events may easily occur in quick succession during the tip of the asymptotic giant branch phases for Main Belt analogues. If this process is common, then we might expect YORP discs to be composed of small (cm-sized or m-sized) monoliths of high internal strength. In Scattered Disc analogues, for which multiple fissions would be less common, the fragmented bodies are more likely to be $\mathrm{km}$-sized rubble piles.

If two terrestrial planets collide with one another at au-scales during the white dwarf phase, the resulting fragments (or planetesimals) could form a different type of disc for which our YORP disc computations would still be applicable. Another possibility is that extant planetesimals could collide with existing terrestrial planets. The resulting impact ejecta may be thrust towards, perturbed close to, or radiatively dragged towards the white dwarf Veras \& Kurosawa 2020). Regardless of how the planetesimals are formed, they would be subject to gravitational perturbations from surviving major planets (Debes \& Sigurdsson 2002; Bonsor et al. 2011; Debes et al. 2012; Veras et al. 2013; Vovatzis et al. 2013; Frewen \& Hansen 2014; Veras \& Gänsicke 2015; Antoniadou \& Veras 2016, 2019; Veras et al. 2016a; Mustill et al. 2018; Smallwood et al. 2018).
Observing the transfer of matter from a YORP disc to a spin or tidal disc is challenging. Current capabilities are limited to detecting dust $\mathrm{Xu}$ et al. 2013; Farihi et al. 2014), although the highly eccentric transiting material (with an eccentricity of 0.97 ) orbiting ZTF J0139+5245 (Vanderbosch et al. 2020) may be indicative of a transfer-inprogress. This progenitor of the observed debris could have broken up due to either rotational fission or tidal disruption depending on the location of its orbital pericentre and its physical shape. If rotational fission is the origin, then subsequent to radiative circularization (Veras et al. 2015b), the debris will form a spin disc.

We have shown here that the survival timescales for spin discs are strongly dependent on the values of $R_{\mathrm{p}}$ and $M_{\mathrm{disc}}$, and can vary from seconds to about 1 Myr (despite some cold disc cases with small numbers of planetesimals where the survival timescales exceed this value). If the disc mass in ZTF J0139+5245 is $10^{14}-10^{15} \mathrm{~kg}$ (Veras et al. 2020a), then Fig. 5 demonstrates that the lifetime of this disc is at least hundreds if not thousands of years; only if the star was brighter would the disc be influenced by the Yarkovsky effect.

Maximum lifetimes of tidal debris discs are a couple of orders of magnitude shorter than those of spin debris discs. Figs. 6 6 17 suggest that 0.01 Myr may be considered as an upper bound for tidal discs before they are sure to break down into dust and/or gas. However, our phase space exploration indicates that most of the tidal discs that we constructed would depart from a steady state within observable timescales of years.

Consequently, detecting photometric curves of just planetesimals - without any broad tails due to dust - would provide particularly valuable evolutionary constraints and spur additional imminent observations. In the case of WD $1145+017$, the disc contains gas, dust and fragments, such that the likely lone progenitor object in that system cannot be thought of to reside in the types of debris discs that we consider here.

However, Veras et al. (2016b) investigated gas-free disc analogues of the WD $1145+017$ planetary system with 4,6 and 8 planetesimals with $M_{\mathrm{p}}=10^{17}-10^{23} \mathrm{~kg}$. They ran $N$ body simulations lasting five years primarily to determine transit timing variations, but also secondarily to determine the instability timescales of these debris discs. Over their simulation duration, their $M_{\text {disc }}=4-8 \times 10^{17} \mathrm{~kg}$ discs remained stable. However, their study is not necessarily comparable to ours because they placed the planetesimals on exactly circular and co-orbital orbits $\left(\sigma_{e}=\sigma_{i}=0\right)$. As we have demonstrated, small eccentricity dispersions on the order of $10^{-5}$ can make a significant difference to $\max \left(t_{\text {disc }}\right)$.

Our computations for $\max \left(t_{\text {disc }}\right)$ assume that the disc is not influenced by external forces. External accretion onto the disc itself from incoming material (Brown et al. 2017) may be common and affect the steady state lifetime (Kenyon \& Bromley 2017a). Further, the timescale on which

4 The standout exceptions are the cold tidal discs with small numbers of large planetesimals. These discs are physically plausible given that, at least in WD $1145+017$, the orbital eccentricity of the progenitor asteroid is probably under $1 \times 10^{-2}$ Gurri et al. 2017; Veras et al. 2017a) 


\section{Collision-limited hot tidal discs}

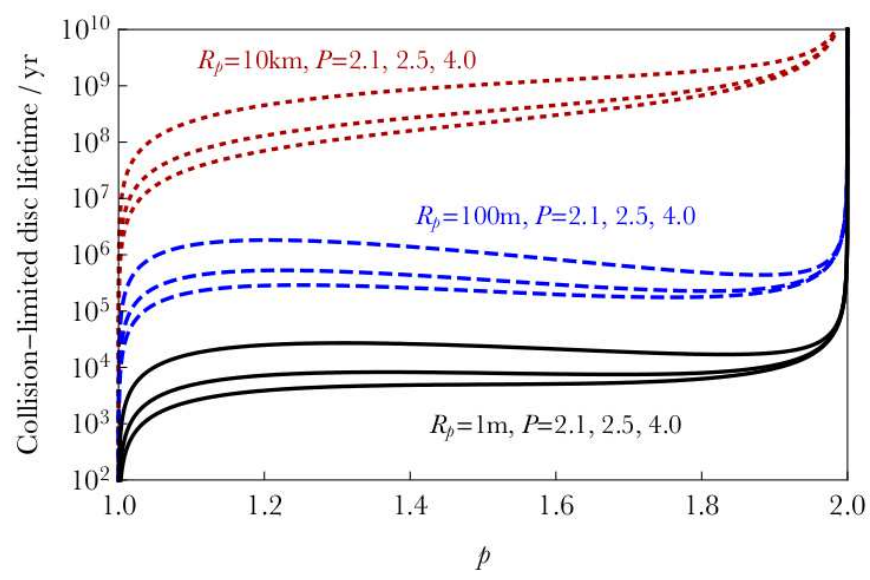

Figure 8. Lifetimes of collision-dominated hot tidal discs $\left[\left(r_{\mathrm{i}}-\right.\right.$ $\left.r_{\mathrm{o}}\right)=(0.6-1.2) R_{\odot}, \sigma_{e}=2 \times 10^{-3}=2 \sigma_{i}, Q_{0}=10^{7} \mathrm{erg} / \mathrm{g}$, $\left.M_{\text {disc }}=10^{20} \mathrm{~kg}, \rho_{\mathrm{p}}=2 \mathrm{~g} / \mathrm{cm}^{3}, M_{\star}=0.6 M_{\odot}\right]$ which are not monodisperse, but rather follow a power-law mass distribution given by Eq. (44). Plotted are different exponents $(p, P)$ for this distribution, as well as three different values of the planetesimal radius $R_{\mathrm{p}}$ corresponding to the maximum planetesimal mass.

major planets perturb planetesimals from the YORP disc close to the white dwarf may be shorter than $\max \left(t_{\text {disc }}\right)$. However, this perturbation timescale is highly model dependent.

Another type of external influence on the disc itself are these major planets. Tidal migration of these planets would disrupt if not destroy extant spin or tidal discs. High-eccentricity (O'Connor \& Lai 2020) and chaotic (Veras \& Fuller 2019) migration could see a planet plough through a white dwarf disc. Slower tidal migration mechanisms that rely on magnetism (Bromley \& Kenyon 2019; Veras \& Wolszczan 2019) and quality function variations (Veras et al. 2019b) could also represent disruptive influences, just on a different timescale.

Even neglecting potential external influences on the discs, one may attempt to link $M_{\text {disc }}$ and $\max \left(t_{\text {disc }}\right)$ for YORP, spin and tidal discs with observed accretion rates onto white dwarf atmospheres. However, there are three crucial missing pieces to this story: the delivery frequency of YORP planetesimals to the vicinity of the white dwarf, the amount of accreted material which bypasses the disc phase entirely (Wvatt et al. 2014; Brown et al. 2017; Turner \& Wvatt 2020), and the timescale for the sublimated gas to accrete onto the white dwarf. These quantities are highly model dependent, and the last is likely to require the application of sophisticated simulations with a coupled treatment of gas and dust (Metzger et al. 2012; Kenvon \& Bromley 2017b).

\subsection{Collision-limited discs}

Our investigation has been limited in many respects so that we could focus on computing steady state debris disc lifetimes. Two of our most significant limitations are (i) our assumption that the discs are monodisperse, and that (ii) our computations do not include the disc's subsequent evolution after leaving the steady state. This evolution would require a dedicated detailed investigation (Bochkarev \& Rafikov 2011; Rafikov 2011a.b; Metzger et al. 2012; Rafikov \& Garmilla 2012; Kenyon \& Bromley 2017a, b; Miranda \& Rafikov 2018) and preferably include the physical processes of fragmentation, erosion, collisions, sublimation, condensation and ultimately accretion onto the white dwarf.

Relaxing the assumption of equal-mass planetesimals would require a different model, one which might not admit a tractable analytical form as in Heng \& Tremaine (2010). However, with the existing model, we can still obtain useful results for dispersion-dominated, hot discs by assuming that they are "collision-limited" with a power-law - rather than a monodisperse - mass distribution. The reason is because an initial power-law mass distribution which produces a collision-limited disc that is dominated by small bodies settles towards a state which mimics a monodisperse system of fragments with mass $M_{\max }$.

Section 4.3 of Heng \& Tremaine (2010) presents a method to compute $M_{\max }$ as an implicit function of time. We have a different goal here of computing $t_{\text {disc }}$. We slightly generalize their treatment by starting with Eq. (22), from which we can now write

$\left(t_{\mathrm{disc}}\right)^{-1}=27.68 \frac{\sigma_{r} \sigma_{i}}{\sigma_{e}}\left(\frac{3 M_{\max }}{4 \pi \rho_{\mathrm{p}}}\right)^{\frac{2}{3}} \int_{M_{\min }}^{M_{\max }} \frac{d n_{0}\left(M_{\mathrm{p}}^{\prime}\right)}{d M_{\mathrm{p}}^{\prime}} d M_{\mathrm{p}}^{\prime}$.

Further, assume that the initial mass distribution in the disc is given by the following broken power-law

$$
\begin{aligned}
& \frac{d n_{0}\left(M_{\mathrm{p}}\right)}{d M_{\mathrm{p}}}=\frac{n_{\max }}{M_{\max }}\left(\frac{M_{\mathrm{p}}}{M_{\max }}\right)^{-p}, \quad p<2 \text { and } M_{\mathrm{p}} \leqslant M_{\max } \\
& \frac{d n_{0}\left(M_{\mathrm{p}}\right)}{d M_{\mathrm{p}}}=\frac{n_{\max }}{M_{\max }}\left(\frac{M_{\mathrm{p}}}{M_{\max }}\right)^{-P}, \quad P>2 \text { and } M_{\mathrm{p}} \geqslant M_{\max }
\end{aligned}
$$

where

$n_{\max }=\frac{\rho_{0}}{M_{\max }}\left(\frac{1}{2-p}+\frac{1}{P-2}\right)^{-1}$

and where the minimum and maximum planetesimal masses in the disc are related through

$M_{\min }=M_{\max } \frac{Q_{\mathrm{D}}^{\star}}{\sigma_{r}^{2}}$.

Integration finally yields

$\left(t_{\mathrm{disc}}\right)^{-1}=27.68 \frac{\sigma_{r} \sigma_{i}}{\sigma_{e}}\left(\frac{3 M_{\max }}{4 \pi \rho_{\mathrm{p}}}\right)^{\frac{2}{3}} \frac{n_{\max }}{p-1}\left(\frac{Q_{\mathrm{D}}^{\star}}{\sigma_{r}^{2}}\right)^{1-p}$

where $Q_{\mathrm{D}}^{\star}$ is a function of $M_{\max }$ through Eq. (29). We can now compute disc lifetime as a function of the steepness of the size distribution, and do plot the result in Fig. 8 The plot illustrates that a uniform decrease in $R_{\mathrm{p}}$ does not correspond to a uniform decrease in $t_{\text {life, }}$, and that $t_{\text {life }}$ is relatively independent of $P$ when $P>2.5$.

\section{SUMMARY}

The evolution timescales of planetary debris discs around white dwarfs represent crucial parameters for our under- 
standing of post-main-sequence planetary science. Here, we analytically computed the maximum steady state lifetimes of three types of monodisperse white dwarf debris discs: those composed of debris from (i) giant branch YORP breakup at $2-100 \mathrm{au}$, (ii) radiation-less rotational fission at $1.5-4.5 R_{\odot}$, and (iii) tidal disruption within $1.3 R_{\odot}$. We displayed a series of figures which cover nearly the entire relevant parameter space, and hence can be used to read off and extrapolate bounds for individual systems or ensembles.

We found that YORP discs of masses ranging from $10^{12}-10^{24} \mathrm{~kg}$ are sufficiently long-lived to provide a reservoir of planetesimals of sizes $10^{-2}-10^{5} \mathrm{~m}$ which can be delivered intact to white dwarfs of any cooling age. The other two types of debris discs which are formed closer to the vicinity of the white dwarf cannot survive in a steady state for longer than about $1 \mathrm{Myr}$ (for the fissional spin discs) and $0.01 \mathrm{Myr}$ (for the tidal discs). However, in the majority of parameter space, these discs leave their steady state within about 1 yr. Hence, transit detections of white dwarf planetesimals without dusty tails should be monitored regularly to detect imminent dynamical activity.

\section{ACKNOWLEDGEMENTS}

We thank the reviewer for their helpful comments, which have improved the manuscript. DV gratefully acknowledges the support of the STFC via an Ernest Rutherford Fellowship (grant ST/P003850/1). KH acknowledges partial financial support from the Center for Space and Habitability (CSH), the PlanetS National Center of Competence in Research (NCCR), the Swiss National Science Foundation, the MERAC Foundation and a European Research Council (ERC) Consolidator Grant (number 771620).

\section{REFERENCES}

Adams, F. C., \& Bloch, A. M. 2013, ApJL, 777, L30 Althaus, L. G., Córsico, A. H., Isern, J., \& García-Berro, E. 2010, ARA\&A, 18, 471

Antoniadou, K. I., \& Veras, D. 2016, MNRAS, 463, 4108

Antoniadou, K. I., \& Veras, D. 2019, A\&A, 629, A126

Benz, W., \& Asphaug, E. 1999, Icarus, 142, 5

Bochkarev, K. V., \& Rafikov, R. R. 2011, ApJ, 741, 36

Bonsor, A., \& Wyatt, M. 2010, MNRAS, 409, 1631

Bonsor, A., Mustill, A. J., \& Wyatt, M. C. 2011, MNRAS, 414, 930

Bonsor, A., \& Veras, D. 2015, MNRAS, 454, 53

Bonsor, A., Farihi, J., Wyatt, M. C., \& van Lieshout, R. 2017, MNRAS, 468, 154

Bonsor, A., Carter, P. J., Hollands, M., et al. 2020, MNRAS, 492, 2683

Bromley, B. C., \& Kenyon, S. J. 2019, ApJ, 876, 17

Brown, J. C., Veras, D., \& Gänsicke, B. T. 2017, MNRAS, 468, 1575

Caiazzo, I., \& Heyl, J. S. 2017, Submitted to MNRAS, arXiv:1702.07682

Cauley, P. W., Farihi, J., Redfield, S., et al. 2018, ApJ, 852, L22.
Cazenave, A., Lago, B., \& Dominh, K. 1982, Icarus, 51, 133

Chambers, J. E., Wetherill, G. W., \& Boss, A. P. 1996, Icarus, 119, 261

Chatterjee, S., Ford, E. B., Matsumura, S., \& Rasio, F. A. 2008, ApJ, 686, 580-602

Cummings, J. D., Kalirai, J. S., Tremblay, P.-E., et al. 2018, ApJ, 866, 21

Debes, J. H., \& Sigurdsson, S. 2002, ApJ, 572, 556

Debes, J. H., Walsh, K. J., \& Stark, C. 2012, ApJ, 747, 148

Dennihy, E., Clemens, J. C., Dunlap, B. H., Fanale, S. M.,

Fuchs, J. T., Hermes, J. J. 2018, ApJ, 854, 40

Dones, L., \& Tremaine, S. 1993, Icarus, 103, 67

Dong, R., Wang, Y., Lin, D. N. C., \& Liu, X.-W. 2010, ApJ, 715, 1036

Doyle, A. E., Young, E. D., Klein, B., et al. 2019, Science, 366,356

Dufour, P., Kilic, M., Fontaine, G., et al. 2010, ApJ, 719, 803

Duvvuri, G. M., Redfield, S., \& Veras, D. 2020, ApJ In Press, arXiv:2003.08410

Farihi, J., Barstow, M. A., Redfield, S., Dufour, P., \& Hambly, N. C. 2010, MNRAS, 404, 2123

Farihi, J., Wyatt, M. C., Greaves, J. S., et al. 2014, MNRAS, 444, 1821

Farihi, J. 2016, New Astronomy Reviews, 71, 9

Farihi, J., van Lieshout, R., Cauley, P. W., et al. 2018, MNRAS, 481, 2601

Frewen, S. F. N., \& Hansen, B. M. S. 2014, MNRAS, 439, 2442

Funk, B., Wuchterl, G., Schwarz, R., et al. 2010, A\&A, 516, A82

Gabriel, T. S. J., Jackson, A. P., Asphaug, E., et al. 2020, ApJ, 892, 40

Gallet, F., Bolmont, E., Mathis, S., Charbonnel, C., \& Amard, L. 2017, In Press A\&A, arXiv:1705.10164

Gänsicke, B. T., Marsh, T. R., Southworth, J., \& RebassaMansergas, A. 2006, Science, 314, 1908

Gänsicke, B. T., Koester, D., Farihi, J., et al. 2012, MNRAS, 424, 333

Gänsicke, B. T., Aungwerojwit, A., Marsh, T. R., et al. 2016, ApJL, 818, L7

Gänsicke, B. T., Schreiber, M. R., Toloza, O., et al. 2019, Nature, 576, 61

Gary, B. L., Rappaport, S., Kaye, T. G., Alonso, R., \& Hambschs, F.-J. 2017, MNRAS, 465, 3267

Girven, J., Brinkworth, C. S., Farihi, J., et al. 2012, ApJ, 749,154

Graham, J. R., Matthews, K., Neugebauer, G., \& Soifer, B. T. 1990, ApJ, 357, 216

Greenzweig, Y., \& Lissauer, J. J. 1992, Icarus, 100, 440

Gurri, P., Veras, D., \& Gänsicke, B. T. 2017, MNRAS, 464, 321

Hadjidemetriou, J. D. 1963, Icarus, 2, 440

Hamers A. S., Portegies Zwart S. F., 2016, MNRAS, 462, L84

Harrison, J. H. D., Bonsor, A., \& Madhusudhan, N. 2018, MNRAS, 479, 3814.

Heng, K., \& Malik, M. 2013, MNRAS, 432, 2562

Heng, K., \& Tremaine, S. 2010, MNRAS, 401, 867

Hollands, M. A., Koester, D., Alekseev, V., Herbert, E. L., \& Gänsicke, B. T. 2017, MNRAS, 467, 4970 
Hollands, M. A., Gänsicke, B. T., \& Koester, D. 2018, MNRAS, 477, 93.

Hornung, P., Pellat, R., \& Barge, P. 1985, Icarus, 64, 295 Izquierdo, P., Rodríguez-Gil, P., Gänsicke, B. T., et al. 2018, MNRAS, 481, 703.

Jura, M. 2003, ApJL, 584, L91

Jura, M. 2008, AJ, 135, 1785

Jura, M., \& Young, E. D. 2014, Annual Review of Earth and Planetary Sciences, 42, 45

Kenyon S. J., Bromley B. C., 2017a, ApJ, 844, 116

Kenyon S. J., Bromley B. C., 2017b, ApJ, 850, 50

Klein, B., Jura, M., Koester, D., Zuckerman, B., \& Melis, C. 2010, ApJ, 709, 950

Koester, D. 2013, Planets, Stars and Stellar Systems. Volume 4: Stellar Structure and Evolution, 4, 559

Kunitomo, M., Ikoma, M., Sato, B., Katsuta, Y., \& Ida, S. 2011, ApJ, 737, 66

Madappatt, N., De Marco, O., \& Villaver, E. 2016, MNRAS, 463, 1040

Makarov, V. V., \& Veras, D. 2019, ApJ, 886, 127

Malamud, U., \& Perets, H. B. 2020a, MNRAS, 492, 5561

Malamud, U., \& Perets, H. B. 2020b, MNRAS, In Press, arXiv:1911.12184

Manser, C. J., Gänsicke, B. T., Marsh, T. R., et al. 2016a, MNRAS, 455, 4467

Manser, C. J., Gänsicke, B. T., Koester, D., et al. 2016b, MNRAS, 462, 1461

Manser, C. J., Gänsicke, B. T., Eggl, S., et al. 2019, Science, 364,66

Manser, C. J., Gänsicke, B. T., Gentile Fusillo, N. P., et al. 2020, MNRAS, 493, 2127

Mestel, L. 1952, MNRAS, 112, 583

Metzger, B. D., Rafikov, R. R., \& Bochkarev, K. V. 2012, MNRAS, 423, 505

Miranda, R., \& Rafikov, R. R. 2018, ApJ, 857, 135.

Mustill, A. J., \& Villaver, E. 2012, ApJ, 761, 121

Mustill, A. J., Veras, D., \& Villaver, E. 2014, MNRAS, 437, 1404

Mustill, A. J., Villaver, E., Veras, D., Gänsicke, B. T., Bonsor, A. 2018, MNRAS, 476, 3939.

Nauenberg, M. 1972, ApJ, 175, 417

Nordhaus, J., \& Spiegel, D. S. 2013, MNRAS, 432, 500

O'Connor, C. E., \& Lai, D. 2020, Submitted to MNRAS, arXiv:2005.05977

Omarov, T. B. 1962, Izv. Astrofiz. Inst. Acad. Nauk. KazSSR, 14, 66

Payne, M. J., Veras, D., Holman, M. J., Gänsicke, B. T. 2016, MNRAS, 457, 217

Payne, M. J., Veras, D., Gänsicke, B. T., \& Holman, M. J. 2017, MNRAS, 464, 2557

Pearce, T. D., \& Wyatt, M. C. 2014, MNRAS, 443, 2541

Petrovich, C., \& Muñoz, D. J. 2017, ApJ, 834, 116

Pu, B., \& Wu, Y. 2015, ApJ, 807, 44

Rafikov, R. R. 2011a, MNRAS, 416, L55

Rafikov, R. R. 2011b, ApJL, 732, L3

Rafikov, R. R., \& Garmilla, J. A. 2012, ApJ, 760, 123

Rao S., et al., 2018, A\&A, 618, A18

Rappaport, S., Gary, B. L., Kaye, T., et al. 2016, MNRAS, 458, 3904

Rappaport, S., Gary, B. L., Vanderburg, A., et al. 2018, MNRAS, 474, 933
Redfield, S., Farihi, J., Cauley, P. W., et al. 2017, ApJ, 839, 42.

Rocchetto, M., Farihi, J., Gänsicke, B. T., et al. 2015, MNRAS, 449, 574

Rogers, L. K., Xu, S., Bonsor, A., et al. 2020, MNRAS In Press, doi:10.1093/mnras/staa873

Ronco, M. P., Schreiber, M. R., Giuppone, C. A., Veras, D., Cuadra, J., Guilera, O. M. 2020, Submitted to ApJL Rubincam, D. P. 2000, Icarus, 148, 2

Scheeres, D. J. 2018, Icarus, 304, 183

Schleicher, D. R. G., \& Dreizler, S. 2014, A\&A, 563, A61

Schröder, K.-P., \& Connon Smith, R. 2008, MNRAS, 386, 155

Smallwood, J. L., Martin, R. G., Livio, M., \& Lubow, S. H. 2018, MNRAS, 480, 57

Smith, A. W., \& Lissauer, J. J. 2009, Icarus, 201, 381

Staff, J. E., De Marco, O., Wood, P., Galaviz, P., \& Passy, J.-C. 2016, MNRAS, 458, 832

Stephan, A. P., Naoz, S., \& Zuckerman, B. 2017, ApJL, 844, L16

Stephan, A. P., Naoz, S., \& Gaudi, B. S. 2018, AJ, 156, 128

Stewart, G. R., \& Ida, S. 2000, Icarus, 143, 28

Stone, N., Metzger, B. D., \& Loeb, A. 2015, MNRAS, 448, 188

Su, K. Y. L., Chu, Y.-H., Rieke, G. H., et al. 2007, ApJL, 657, L41

Sun M., Arras P., Weinberg N. N., Troup N. W., Majewski S. R., 2018, MNRAS, 481, 4077

Swan, A., Farihi, J., \& Wilson, T. G. 2019a, MNRAS, 484, L109

Swan, A., Farihi, J., Koester, D., et al. 2019b, MNRAS, 490, 202

Toomre, A. 1964, ApJ, 139, 1217

Tremblay P.-E., Cummings J., Kalirai J. S., Gänsicke B. T., Gentile-Fusillo N., Raddi R., 2016, MNRAS, 461, 2100

Turner, S. G. D., \& Wyatt, M. C. 2020, MNRAS, 491, 4672 van Lieshout, R., Kral, Q., Charnoz, S., et al. 2018, MNRAS, 480, 2784.

van Maanen, A. 1917, PASP, 29, 258

van Maanen, A. 1919, AJ, 32, 86

Vanderbosch, Z., Hermes, J. J., Dennihy, E., et al. 2020, In Press ApJ, arXiv: 1908.09839

Vanderburg, A., Johnson, J. A., Rappaport, S., et al. 2015, Nature, 526, 546

Vanderburg, A., \& Rappaport, S. A. 2018, Handbook of Exoplanets, 37, Eds: Hans J. Deeg, Juan Antonio Belmonte. Springer International Publishing

Veras, D., Wyatt, M. C., Mustill, A. J., Bonsor, A., \& Eldridge, J. J. 2011, MNRAS, 417, 2104

Veras, D., \& Wyatt, M. C. 2012, MNRAS, 421, 2969

Veras, D., Mustill, A. J., Bonsor, A., \& Wyatt, M. C. 2013, MNRAS, 431, 1686

Veras, D., Leinhardt, Z. M., Bonsor, A., Gänsicke, B. T. 2014a, MNRAS, 445, 2244

Veras, D., Jacobson, S. A., Gänsicke, B. T. 2014b, MNRAS, 445,2794

Veras, D., Shannon, A., Gänsicke, B. T. 2014c, MNRAS, 445,4175

Veras, D., Evans, N. W., Wyatt, M. C., \& Tout, C. A. 2014d, MNRAS, 437, 1127

Veras, D., \& Gänsicke, B. T. 2015, MNRAS, 447, 1049 
Veras, D., Eggl, S., Gänsicke, B. T. 2015a, MNRAS, 451, 2814

Veras, D., Leinhardt, Z. M., Eggl, S., Gänsicke, B. T. 2015b, MNRAS, 451, 3453

Veras, D. 2016a, Royal Society Open Science, 3, 150571

Veras, D. 2016b, MNRAS, 463, 2958

Veras, D., Mustill, A. J., Gänsicke, B. T., et al. 2016a, MNRAS, 458, 3942

Veras, D., Marsh, T. M., Gänsicke, B. T. 2016b, MNRAS, 461, 1413

Veras, D., Carter, P. J., Leinhardt, Z. M., \& Gänsicke, B. T. 2017a, MNRAS, 465, 1008

Veras, D., Georgakarakos, N., Dobbs-Dixon, I., \& Gänsicke, B. T. 2017b, MNRAS, 465, 2053

Veras D., Georgakarakos N., Gänsicke B. T., Dobbs-Dixon I., 2018, MNRAS, 481, 2180

Veras, D., \& Fuller, J. 2019, MNRAS, 489, 2941

Veras, D., \& Wolszczan, A. 2019, MNRAS, 488, 153

Veras, D., Higuchi, A., \& Ida, S. 2019a, MNRAS, 485, 708

Veras, D., Efroimsky, M., Makarov, V. V., et al. 2019b, MNRAS, 486, 3831

Veras, D. 2020, MNRAS, 493, 4692

Veras, D., \& Fuller, J. 2020, MNRAS, 492, 6059

Veras, D., \& Kurosawa, K. 2020, MNRAS, 494, 442

Veras, D., \& Scheeres, D. J. 2020, MNRAS, 492, 2437

Veras, D., McDonald, C. H., \& Makarov, V. V. 2020a, MNRAS, 492, 5291

Veras, D., Reichert, K., Flammini Dotti, F., et al. 2020b, MNRAS, doi:10.1093/mnras/staa559

Verbunt, F., \& Rappaport, S. 1988, ApJ, 332, 193

Villaver, E., Livio, M., Mustill, A. J., \& Siess, L. 2014, ApJ, 794, 3

Völschow, M., Banerjee, R., \& Hessman, F. V. 2014, A\&A, 562, A19

Voyatzis, G., Hadjidemetriou, J. D., Veras, D., \& Varvoglis, H. 2013, MNRAS, 430, 3383

Wang, T.-. gui ., Jiang, N., Ge, J., et al. 2019, ApJL, 886, L5

Wilson, D. J., Gänsicke, B. T., Koester, D., et al. 2014, MNRAS, 445, 1878

Wilson, D. J., Gänsicke, B. T., Koester, D., et al. 2014, MNRAS, 445, 1878

Wilson, T. G., Farihi, J., Gänsicke, B. T., et al. 2019, MNRAS, 487, 133

Wyatt, M. C., Farihi, J., Pringle, J. E., \& Bonsor, A. 2014, MNRAS, 439, 3371

Xu, S., \& Jura, M. 2012, ApJ, 745, 88

$\mathrm{Xu}$, S., Jura, M., Klein, B., Koester, D., \& Zuckerman, B. 2013, ApJ, 766, 132

Xu, S., \& Jura, M. 2014, ApJL, 792, L39

Xu, S., Rappaport, S., van Lieshout, R., et al. 2018a, MNRAS, 474, 4795

Xu, S., Su, K. Y. L., Rogers, L. K., et al. 2018b, ApJ, 866, 108

Xu, S., Hallakoun, N., Gary, B., et al. 2019, AJ, 157, 255

Zhou, J.-L., Lin, D. N. C., \& Sun, Y.-S. 2007, ApJ, 666, 423

Zotos, E. E.. Veras. D. 2020, In Press A\&A, 637, A14

Zuckerman, B., \& Becklin, E. E. 1987, Nature, 330, 138

Zuckerman, B., Koester, D., Melis, C., Hansen, B. M., \& Jura, M. 2007, ApJ, 671, 872 